NBER WORKING PAPER SERIES

\title{
ILLIQUID ASSETS AND OPTIMAL PORTFOLIO CHOICE
}

\author{
Eduardo S. Schwartz \\ Claudio Tebaldi \\ Working Paper 12633 \\ http://www.nber.org/papers/w12633 \\ NATIONAL BUREAU OF ECONOMIC RESEARCH \\ 1050 Massachusetts Avenue \\ Cambridge, MA 02138 \\ October 2006
}

The present paper has been started while the author was member of the faculty of Economics, Verona University and visiting scholar at the Anderson School of Management, Faculty of Finance. We thank J. Cvitanic for his helpful comments on an earlier version of the paper. C.T. gratefully acknowledges the Anderson School, the Finance Faculty and in particular Prof. Schwartz for the kind invitation and warm hospitality. C.T. would also like to thank Marina Di Giacinto for constant support and many valuable comments, Martino Grasselli, the participants to the seminar at University of Torino, to the workshop at Bocconi University, R. Gibson, F.Gozzi, F. Zapatero and the participants to the WFA 2007 meeting for several insights and helpful discussions. The views expressed herein are those of the author(s) and do not necessarily reflect the views of the National Bureau of Economic Research.

(C) 2006 by Eduardo S. Schwartz and Claudio Tebaldi. All rights reserved. Short sections of text, not to exceed two paragraphs, may be quoted without explicit permission provided that full credit, including (C) notice, is given to the source. 
Illiquid Assets and Optimal Portfolio Choice

Eduardo S. Schwartz and Claudio Tebaldi

NBER Working Paper No. 12633

October 2006Ш5 HMLHG1 RYP EHU प००

JEL No. G11

\begin{abstract}
$\underline{\text { ABSTRACT }}$
The presence of illiquid assets, such as human wealth or a family owned business, complicates the problem of portfolio choice. This paper is concerned with the problem of optimal asset allocation and consumption in a continuous time model when one asset cannot be traded. This illiquid asset, which depends on an uninsurable source of risk, provides a liquid dividend. In the case of human capital we can think about this dividend as labor income. The agent is endowed with a given amount of the illiquid asset and with some liquid wealth which can be allocated in a market where there is a risky and a riskless asset. The main point of the paper is that the optimal allocations to the two liquid assets and consumption will critically depend on the endowment and characteristics of the illiquid asset, in addition to the preferences and to the liquid holdings held by the agent. We provide what we believe to be the first analytical solution to this problem when the agent has power utility of consumption and terminal wealth. We also derive the value that the agent assigns to the illiquid asset. The risk adjusted valuation procedure we develop can be used to value both liquid and illiquid assets, as well as contingent claims on those assets.
\end{abstract}

Eduardo S. Schwartz

Anderson Graduate School of Management

UCLA

110 Westwood Plaza

Los Angeles, CA 90095

and NBER

eduardo.schwartz@anderson.ucla.edu

Claudio Tebaldi

Dipartimento di Scienze Economiche

Sezione Giardino Giusti

Via Giardino Giusti 2

37129 Verona , Italy

claudio.tebaldi@mail.univr.it 


\section{Introduction}

The problem of optimal asset allocation is of great importance both in the theory as well as in the practice of finance. Since the seminal work of Markowitz (1952) scholars and practitioners have looked at the issue of how much money should an investor optimally allocate to different assets or asset classes. The single period model of Markowitz (1952) was extended to a multiperiod setting by Samuelson (1969) and then to continuous time by Merton $(1969,1971)$. The traditional approach assumes that all assets can be traded at all times. This paper is concerned with optimal asset allocation in a continuous time model when one asset cannot be traded.

Typical examples of assets in which trading is problematic include human wealth and a family owned business. When the asset allocation problem is solved without taking into account the existence of these "illiquid" assets the allocation is certainly suboptimal. Consider the following example. ${ }^{\text {a }}$ Two individuals with the same wealth, the same preferences and the same horizon would invest in the same portfolio using the traditional asset allocation framework. However, if one of the individuals is a stock broker with his human wealth highly correlated with the stock market, and the other is a tenured university professor with his human wealth independent of the stock market, it would be reasonable to expect that they would have different allocations. This is the problem we address in this paper.

There are many definitions of illiquid assets. To make the problem tractable, in this paper we assume that illiquidity prevents the trading of the asset over the time horizon we consider (though this time horizon could become infinite). The illiquid asset, however, provides a liquid "dividend" that is related to the level of an observable state variable associated with the illiquid asset. In the case of human wealth the dividend could be labor income and in the case of a proprietorship it could be distributed profits from the business. The uncertainty that drives the illiquid asset cannot be fully diversified in the market. Since the asset is not traded, the state variable associated with the illiquid asset can not be interpreted as a price. In the finite horizon case this state variable becomes the price of the asset only at the terminal date.

We assume that the agent in endowed with a given amount of the illiquid asset and with some liquid wealth which can be allocated in a market where there are two liquid assets: a risky asset and a risk free asset. The main point of this paper is that the allocations to the two liquid assets and consumption will critically depend on the endowment and characteristics of the illiquid asset, in addition to the preferences and liquid wealth of the agent.

\footnotetext{
aThis example was presented by Robert Merton in a talk in Verona, Italy in June 2003.
} 
In the process of establishing the optimal allocation to the liquid assets we also derive the value that the agent assigns to the illiquid asset. This value depends not only on the level of the state variable associated with the illiquid asset, but also on the preferences of the agent.

The problem of asset allocation in the presence of illiquid assets has been the subject of intense research in the finance literature since the late 60's. Recognizing the complexity of the subject simplifying assumptions have been introduced to make the problem tractable.

Among non tradable assets, human wealth is certainly the most relevant source of risk in the individual allocation problem which is difficult to insure or diversify. Bodie, Merton and Samuleson (1992) consider a long horizon investor with a riskless stream of labor income and show that an investor with riskless non tradable human wealth should tilt his financial portfolio toward stocks relative to an investor who owns only tradable stock. Jagannathan and Kocherlakota (1996) show that this advice is economically sound as long as the human wealth is relatively uncorrelated with stock returns. Zeldes (1989) performs a numerical study of a discrete time model of optimal consumption in the presence of stochastic income. Koo (1995) and Heaton and Lucas (1997) introduce risky labor income and portfolio constraints in an infinite horizon portfolio choice problem and, using a numerical simulation, focus on how the presence of background risks from sources such as labor, influences consumption and portfolio choice. Both papers find that investors hold most of their financial wealth in stocks. Koo (1995) shows numerically that an increase in the variance of permanent income shocks decreases both the optimal portfolio allocation to stocks and the consumption labor income ratio of power utility investors. In a discrete time framework Viceira (2001) considers an approximate solution and finds that positive correlation between labor income innovations and unexpected financial returns reduces the investor's willingness to hold liquid risky asset because of its poor properties as an hedge against unexpected declines in labor income. Consistently, Heaton and Lucas (2000) find that entrepreneurs have significantly safer portfolios of financial assets than other investors with similar wage and wealth. Campbell and Viceira (2002) provide a comprehensive discussion of the empirical testing and of the economic implications of including human wealth in the household portfolio choice problem. Dybvig and Liu (2004) consider a lifetime consumption/investment model with endogenous retirement date. Wang (2006) provides a complete discussion of the consumption choices of an agent with human and liquid wealth under the assumption of exponential utility function.

A related literature deals with portfolio choice in the presence of assets which cannot be traded. To our knowledge the first treatment is due to Myers 
$(1972,1973)$ which solves the static version of the problem. In a dynamic context, the problem we solve can be seen as the limit of large transaction costs of the Grossmann and Laroque (1990) model for illiquid durables. Svensson and Werner (1993) provide a treatment with exponential preferences. Kahl Liu and Longstaff (2003) formulate and provide a numerical solution to the optimal dynamic allocation problem of an investor with power utility whose portfolio includes a stock which cannot be sold. Among the possible sources of background risk in household portfolios, housing is certainly one important asset class that is relatively illiquid and undiversified. Analyzing risk and return is however complicated because of the unobservable flow of consumption of housing services. Flavin and Yamashita (1998) consider housing both as an asset and as a source of consumption, and obtain the optimal portfolio allocations by simulation.

There is a large strand of the literature in stochastic optimization which addresses the continuous time portfolio allocation problem in incomplete markets both with the direct partial differential equation approach and with the martingale-measure duality approach. Duffie, Fleming, Soner and Zariphopolou (1997) study an asset allocation problem for an investor which maximizes HARA utility (with relative risk aversion coefficient smaller than 1) from consumption in a market composed by a risky and a riskless asset and receives an income which cannot be replicated by other securities. This study proves existence, uniqueness and regularity of the value function, while the optimal consumption path and the allocation strategy are implicitly specified throughout a feedback expression. Koo (1998) analyzes the same problem in the presence of constraints.

The stochastic optimization problem we discuss is strictly related to the utility based pricing of contingent claims whose underlying assets are non traded. Some of the important references are: Davis (1999), Detemple and Sundaresan (1999), Teplà (2000), Hobson and Henderson (2002), Henderson (2002) and Musiela and Zariphopolou (2004a). However, the case of an agent with power utility for consumption and terminal wealth, with dividends from the illiquid asset has so far resisted analytical treatment.

Our results are based on the duality approach pioneered by Cox and Huang (1989), He and Person (1991), and Karatzas et al. (1991). He and Pages (1993) and El Karoui and Jeanblanc (1998) deal with a constrained version of the problem when labor income risk can be diversified in the market. When the agent receives an uninsurable random endowment the mathematical formulation of the stochastic control problem becomes difficult. Existence results under very general conditions on the price processes and on the utility function have been obtained by Cuoco (1997) attacking directly the primal problem, while exact results on the duality approach have been established 
by Cvitanic, Schachermayer and Wang (2001) in the case of maximization of utility from terminal wealth and extended by Karatzas and Zitkovic (2003) and Zitkovic (2005) to the problem with intertemporal consumption and constraints. Our solution strategy is closely related to the dual verification approach of Mnif and Pham (2001) the solution to the primal problem is characterized in terms of the solution to a dual singular control problem which is solved by making use of the dynamic programming principle.

As far as we are aware, our paper contains the first analytical solution to this problem when the agent has power utility of consumption and terminal wealth. The analytical solution obtained allows us to quantify the impact of the assets characteristics and the agent preferences on optimal asset allocation and consumption. In particular, we show that the higher is the correlation between the liquid and the illiquid asset, the lower will be the allocation to the risky liquid asset. So, in the example given above the professor would optimally invest a higher proportion of his liquid wealth in the risky liquid asset than the stock broker. Since the human wealth of the stock broker is highly correlated with the stock market, and his human wealth is non tradable, he will tend to invest a smaller fraction of his liquid wealth in the market portfolio.

The analysis of the optimal consumption out of liquid assets shows that the agent will make his consumption decision looking not only at his liquid wealth but taking into account also his illiquid wealth. The computation of the elasticity of consumption with respect to liquid and illiquid wealth shows that for an agent with constant relative risk aversion the propensity to consume out of liquid wealth will be always larger than out of illiquid wealth.

Moreover, the solution provides the expression for the stochastic discount factor that the agent uses for his/her valuation of the illiquid asset. The procedure reduces to risk neutral valuation for the liquid assets, while the risk adjustment for valuing illiquid assets is found to be state dependent. The optimal value function of the allocation/consumption problem determines the analytical expression of the risk adjustment as a function of the liquidity state variable, the preferences of the investor and the volatility of the non-traded source of risk.

The paper is organized as follows. In Section 2 we introduce the economic setting and its mathematical formulation, in Section 3 we provide the analytical solution to the Hamilton Jacobi Bellman equation. Section 4 provides a discussion of the optimal consumption and allocation policies. Section 5 derives the stochastic discount factor that the agent will use in the valuation of the illiquid asset. In Section 6 we analyze the sensitivity of the control policies to different parameters and Section 7 concludes. The Appendix contains the proofs and some technical results. 


\section{Economic setting}

Consider a continuous time economy where prices evolve stochastically in a filtered probability space $\{\Omega, \mathcal{F}, \mathbb{P}\}$ supporting a two dimensional Brownian motion $\left(W_{t}^{1}, W_{t}^{2}\right)$ where $\mathcal{F}=\left\{\mathcal{F}_{t}\right\}_{t<T}$ and $\mathcal{F}_{t}$ represents the augmented filtration generated by all the information reflected in the market up to time $t$ and $\mathbb{P}$ is the objective probability measure. All the processes will be assumed to be adapted to $\mathcal{F}$. We fix a final time horizon $T$, the epoch at which the non traded (illiquid) asset becomes tradable and can be consumed.

The market is composed of three assets:

- The risk free bond $B_{t}$, whose dynamics is:

$$
d B_{t}=r B_{t} d t \quad t \leq T
$$

where $r$ is the continuously compounded risk free interest rate which, for simplicity, we assume to be constant.

- A traded liquid risky asset $S_{t}$, whose dynamics is:

$$
\frac{d S_{t}}{S_{t}}=\alpha d t+\sigma d W_{t}^{1} \quad t \leq T
$$

where $\alpha(>r)$ is the continuously compounded expected rate of return on the risky liquid asset, and $\sigma$ is the continuous standard deviation of the rate of return. ${ }^{\mathrm{b}}$

- An illiquid risky asset $H_{t}$ (no trading is allowed until time $T$, when it can be consumed), whose dynamics is:

$$
\frac{d H_{t}}{H_{t}}=(\mu-\delta) d t+\eta\left(\rho d W^{1}+\sqrt{1-\rho^{2}} d W^{2}\right) \quad t \leq T
$$

where $\mu$ is the continuously compounded total expected rate of return on the risky illiquid asset, $\delta>0$ is the liquid continuous rate of dividend paid by the illiquid asset, $\eta$ is the continuous standard deviation of the rate of return, and $\rho$ is the correlation coefficient between the dynamics of the liquid and the illiquid risky asset. ${ }^{\mathrm{c}}$

\footnotetext{
${ }^{\mathrm{b}}$ For simplicity we assume that the liquid risky asset pays no dividends, but the analysis would be the same if the asset paid a continuous dividend

${ }^{\mathrm{c}}$ Since human wealth will enter in the state equation for liquid wealth equation only through dividends, the dividend plays exactly the same role of a stochastic income for the agent. For this reason, our allocation problem can be considered as the finite horizon counterpart of an allocation problem in the presence of an uninsurable stochastic income.
} 
Since the illiquid asset $H_{t}$ cannot be traded at any time $t<T$, it represents the level of a state variable associated with the illiquid asset, i.e. the process which generates the random cashflows: $\delta H_{t}$ at time $t<T$ and $H_{T}$ at time $T$. At time $T$, the state variable, $H_{T}$, becomes equal to the price of the illiquid asset.

We assume that the investor has time additive separable preferences and maximizes a CRRA utility from consumption and final wealth, with positive relative risk aversion coefficient $\gamma>0$ and different from 1 . The intertemporal optimization problem of the agent is then given by:

$$
\begin{gathered}
\sup _{(\pi, c) \in A(l, h, t)} \mathcal{U}\left(t, c, W_{T}^{\pi, c}\right) \\
\mathcal{U}\left(t, c, W_{T}^{\pi, c}\right):=E_{t}^{\mathbb{P}}\left[\int_{t}^{T} e^{-\kappa(u-t)} U_{\gamma}\left(c_{u}\right) d u+\beta e^{-\kappa(T-t)} U_{\gamma}\left(W_{T}^{\pi, c}\right)\right] \\
\text { with } \quad U_{\gamma}(x)=\frac{x^{1-\gamma}}{1-\gamma} \quad \text { for } x \geq 0, \quad U_{\gamma}(x)=-\infty \quad \text { for } x<0 .
\end{gathered}
$$

The set of admissible plans with initial liquid wealth $l$, and initial level of the illiquid state variable $h, \mathcal{A}(l, h, t)$, is defined as the set of admissible consumption-allocation plans $(c, \pi)$. The consumption stream, $c \equiv\left(c_{\tau}\right)_{t \leq \tau \leq T}$, specifies the agent's consumption rate of liquid assets, while the allocation strategy $\pi=\left(\pi_{\tau}^{S}, \pi_{\tau}^{B}\right)_{t \leq \tau \leq T}$ denotes the dollar amounts invested in the risky and riskless liquid assets at any time $\tau$ between $t$ and $T$. Assume that at time $t$ the agent holds an amount of liquid wealth $l>0$, and a nominal amount of illiquid asset $H_{t}=h \geq 0$, then $(c, \pi)$ is admissible if there exists a strategy $\pi$ which finances a consumption stream $c$, i.e.

$$
\begin{aligned}
L_{t} & =l, \\
L_{\tau} & =\pi_{\tau}^{S}+\pi_{\tau}^{B}, \quad t \leq \tau<T, \\
L_{T} & =\pi_{T}^{S}+\pi_{T}^{B}+H_{T},
\end{aligned}
$$

and the dynamics of liquid wealth is:

$$
d L_{\tau}=\left(r L_{\tau}+\delta H_{\tau}-c_{\tau}\right) d \tau+\pi_{\tau}^{S}\left(\frac{d S_{\tau}}{S_{\tau}}-r d \tau\right)
$$

At the final time $T$ the investor will optimally consume his total liquid wealth, $W_{T}^{\pi, c}=L_{T}$ which includes the wealth obtained by liquid investment, $L_{T^{-}}=$ $\lim _{t \rightarrow T^{-}} L_{t}$, and the illiquid wealth, $H_{T}$, i.e. $L_{T}=L_{T^{-}}+H_{T}$. It is assumed that the agent faces the solvency constraint that the final liquid wealth must be non negative, i.e. $W_{T}^{\pi, c} \geq 0$. If the illiquid asset is perfectly correlated with the market the agent will effectively borrow since he can perfectly hedge the risk of both assets. Our discussion will focus to the more interesting situation 
of an illiquid asset which is partially correlated with the market. It will be assumed that $-1<\rho<1$ and $\gamma>0$. Under these hypotheses, starting with strictly positive liquid wealth, it will never be optimal to reach negative liquid wealth at $t<T$. This is implied by the following argument: suppose on the contrary that a negative position in liquid wealth is possible, then there would be a small but non vanishing probability for the final total wealth to be negative but this is prevented by the fact that marginal utility at time $T$ is diverging at zero liquid wealth. Hence in order to have nonnegative liquid wealth at time $T$ the agent will never borrow and the solvency constraint implies the stronger no-borrowing constraint: $L_{t} \geq 0$ a.s. for any $t \leq T$.

In order to find an optimal solution process for wealth which does not violate the no borrowing constraint, we are forced to enlarge the class of admissible dynamic allocation policies including also singular consumption and allocation policies, i.e. the consumption rate and the allocation can be changed by a finite amount in an infinitesimal time. These singular policies will typically occur when the zero wealth boundary is hit and the liquidity constraint becomes active.

\section{Solution of the stochastic optimization problem}

We search for the value function defined by:

$$
V(l, h, t):=\sup _{(\pi, c) \in A(l, h, t)} \mathcal{U}\left(t, c, W_{T}^{\pi, c}\right) .
$$

The Hamilton Jacobi Bellman (HJB) equation corresponding to the above stochastic optimization problem when $t<T$ can be written as:

$$
\begin{aligned}
0= & V_{t}(l, h, t)+\kappa V_{t}(l, h, t)+\frac{1}{2} \eta^{2} h^{2} V_{h h}(l, h, t)+(r l+\delta h) V_{l}(l, h, t(6) \\
& +(\mu-\delta) h V_{h}(l, h, t)+\max _{\pi^{S}} G\left[\pi^{S}\right]+\max _{c^{\prime} \geq 0} H\left[c^{\prime}\right] \\
G[\pi]= & \frac{1}{2}\left(\pi^{S}\right)^{2} \sigma^{2} V_{l l}(l, h, t)+\pi^{S} \eta \rho \sigma h V_{l h}(l, h, t)+(\alpha-r) \pi^{S} V_{l}(l, h, t) \\
H[c]= & -c V_{l}(l, h, t)+\frac{c^{1-\gamma}}{1-\gamma},
\end{aligned}
$$

when $l, h \geq 0$. With terminal boundary condition:

$$
\lim _{t \rightarrow T^{-}} V(l, h, t)=\beta{\frac{(l+h)^{1-\gamma}}{1-\gamma}}^{1-\gamma}
$$


When $l>0$ the optimal allocation and consumption strategies will be obtained in feedback form solving:

$$
\begin{aligned}
c_{*}(l, h, t) & =\arg \max _{c \geq 0} H(c) \\
& =\left[V_{l}(l, h, t)\right]^{-1 / \gamma} \\
\pi_{*}^{S}(l, h, t) & =\arg \max _{\pi^{S}} G\left(\pi^{S}\right) \\
& =-\frac{\alpha-r}{\sigma^{2}} \frac{V_{l}(l, h, t)}{V_{l l}(l, h, t)}-\frac{\eta \rho}{\sigma} h \frac{V_{l h}(l, h, t)}{V_{l l}(l, h, t)}
\end{aligned}
$$

At zero liquid wealth, $l=0$, the optimal feedback policies are determined by:

$$
\begin{aligned}
& c_{*}^{B}(0, h, t)=\arg \max _{0 \leq c \leq \delta h} H(c) \\
& \pi_{*}^{S}(0, h, t)=0
\end{aligned}
$$

in fact for any allocation to the risky asset different from zero there would be a positive probability to violate the no-borrowing constraint.

\subsection{Homogeneity transformation}

In order to reduce the number of state variables in the HJB equation from two to one we conjecture that the value function that solves eq. (6) when $l \geq 0$ and $h \neq 0$ has the form:

$$
\begin{aligned}
V(l, h, t) & =h^{1-\gamma} V(z, t) \\
z & =l / h,
\end{aligned}
$$

and verify that we obtain a single variable HJB equation for the reduced value function $V(z, t)$. The value function for the problem $h=0$ is given by the solution to the standard Merton (1971) solution. Inserting the expression $(10)$ in $(6)$ and performing the maximizations in $(7,8)$ we obtain that $V(z, t)$, $z>0, t<T$ has to obey: ${ }^{\mathrm{d}}$

$$
\begin{aligned}
0= & V_{t}(z, t)+K_{1} V(z, t)+K_{2} z V_{z}(z, t)-K_{3} \frac{\left(V_{z}(z, t)\right)^{2}}{V_{z z}(z, t)}+ \\
& +K_{4} z^{2} V_{z z}(z, t)+F\left(V_{z}(z, t)\right)
\end{aligned}
$$

where:

$$
F(x)=\max _{c \geq 0}\left\{(-c+\delta) x+\frac{c^{1-\gamma}}{1-\gamma}\right\} \quad \text { if } z>0
$$

\footnotetext{
dThe expressions for the derivatives are given in Appendix 8.9.
} 
and the coefficients are given by:

$$
\begin{aligned}
K_{1} & =-\kappa+(\mu-\delta)(1-\gamma)-\frac{1}{2}(1-\gamma) \gamma \eta^{2} \\
K_{2} & =-(\mu-\delta)+r+(\alpha-r) \frac{\eta \rho}{\sigma}+\gamma \eta^{2}\left(1-\rho^{2}\right) \\
K_{3} & =\frac{1}{2}\left(\frac{(\alpha-r)}{\sigma}-\eta \rho \gamma\right)^{2} \\
K_{4} & =\frac{1}{2} \eta^{2}\left(1-\rho^{2}\right) .
\end{aligned}
$$

When $z=0$ the corresponding reduced HJB equation is given by:

$$
\begin{aligned}
0 & =V_{t}(0, t)+K_{1} V(0, t)+F_{0}\left(V_{z}(0, t)\right), \\
F_{0}(x) & =\max _{0 \leq c \leq \delta}\left\{(-c+\delta) x+\frac{c^{1-\gamma}}{1-\gamma}\right\}
\end{aligned}
$$

The computation of the constrained maximization is detailed in the Appendix. The terminal boundary condition is given by:

$$
V(z, T)=\beta \frac{(1+z)^{1-\gamma}}{1-\gamma}, \quad z=l / h
$$

In a related framework, Duffie et al. (1997) observe that the above reduced HJB equation corresponds to a stochastic optimization problem for an agent with HARA utility solving a consumption investment problem where the state variable $z_{t}$ represents the liquid wealth of the agent at time $t$ which consumes $c \triangleq c_{*}(z, 1, t)$, receives a constant liquid endowment with a rate $\delta$ for $t \leq T$ and a lump sum payment of 1 at time $T$ and the allocation strategies are restricted by the no borrowing constraint $z_{t} \geq 0$. A key difference with respect to the original problem is that the endowment received by the agent is non stochastic, since the change of variables allows to factor the term depending from $H_{t}$ out of the value function. In the next section we use a duality transformation to determine an explicit expression of the reduced value function $V(z, t)$ for $z \geq 0,0 \leq t \leq T$.

\subsection{Duality Transformation}

In order to solve the above HJB equation for $V(z, t)$ we need to consider a change of variables: we introduce the dual value function for the consumption investment problem, $\widetilde{V}(y, t)$, which is related to $V(z, t)$ by the transform:

$$
\widetilde{V}(y, t):=\sup _{z \geq 0}\{V(z, t)-z y\}
$$


The new variable $y$ can be interpreted as the marginal indirect utility level rescaled by a factor $h^{-\gamma}$. The time $T$ terminal condition for the reduced dual value function is given by:

$$
\begin{aligned}
\widetilde{V}(y, T) & =y-\beta^{1 / \gamma} \frac{y^{b}}{b} \quad y \in \mathcal{D}_{T} \triangleq(0, \beta) \\
\widetilde{V}(y, T) & =\frac{\beta}{1-\gamma} \quad y \in \mathcal{D}_{T}^{c} \triangleq[\beta,+\infty) \\
b & =1-\frac{1}{\gamma}
\end{aligned}
$$

The presence of the state constraint complicates the discussion of the dual problem introducing a boundary in the domain of the dual variable $y$.

Under the hypothesis that $\delta>0$, the agent with zero liquid wealth can still consume a fraction of the dividend rate and correspondingly the level of the dual variable, the scaled marginal utility from optimal consumption at the zero wealth boundary is finite, i.e.

$$
y_{B}(t) \triangleq \lim _{z \rightarrow 0^{+}} V_{z}(z, t)<+\infty
$$

thus at each time for $t<T$ the domain of definition of the dual value function can be split into two regions $\mathcal{D}_{t}$ and $\mathcal{D}_{t}^{c}$ defined by

$$
\mathcal{D}_{t} \triangleq\left\{y \in(0,+\infty) \mid y<y_{B}(t)\right\}
$$

and:

$$
\mathcal{D}_{t}^{c} \triangleq\left\{y \in(0,+\infty) \mid y \geq y_{B}(t)\right\} \cap\left\{y \in(0,+\infty) \mid y^{-1 / \gamma} \leq \delta\right\}
$$

The definition of $\mathcal{D}_{t}^{c}$ takes into account that at zero liquid wealth the agent cannot consume more than the liquid dividend rate, hence the set of admissible values for the dual state variable $y \geq y_{B}(t)$ is further restricted by the control constraint $c=y^{-1 / \gamma} \leq \delta$ which implies $y \geq \delta^{-\gamma}$.

Two different domain configurations are possible : if $y_{B}(t) \geq \delta^{-\gamma}$, then $\mathcal{D}_{t}^{c}=\left[y_{B}(t),+\infty\right)$, the set $\mathcal{D}_{t} \cup \mathcal{D}_{t}^{c}=\left(0,+y_{B}(t)\right) \cup\left[y_{B}(t),+\infty\right)$ is connected and $y_{B}(t)$ separates $\mathcal{D}_{t}$ from $\mathcal{D}_{t}^{c}$, i.e. $\overline{\mathcal{D}_{t}} \cap \overline{\mathcal{D}_{t}^{c}}=\left\{y_{B}(t)\right\}$. This situation will be denoted as Regime $I$.

On the contrary, if $y_{B}(t)<\delta^{-\gamma}$ then $\mathcal{D}_{t}^{c}=\left[\delta^{-\gamma},+\infty\right)$, the set $\mathcal{D}_{t} \cup \mathcal{D}_{t}^{c}=$ $\left(0,+y_{B}(t)\right) \cup\left[\delta^{-\gamma},+\infty\right)$ is not connected. This situation will be denoted as Regime $I I$. Its characterizing feature is the anomalous geometry of the state space, which is formed by two regions not connected. 


\subsection{The dual value function in $\mathcal{D}_{t}$}

The reconstruction of the scaled primal value functions from the dual one is straightforward in the domain $\mathcal{D}_{t}$ provided that the dual value function verifies the following assumption on regularity:

Condition A The reduced dual value function $\widetilde{V}(y, t)$ is a strictly convex, continuous function with continuous derivatives w.r.t to $y \in \mathcal{D}_{t}$ up to third order and continuous first order derivatives w.r.t. $t \in(0, T) . \widetilde{R}(y, t) \triangleq$ $-\widetilde{V}_{y}(y, t) /\left[y \widetilde{V}_{y y}(y, t)\right]$ is bounded, i.e. $|\widetilde{R}(y, t)| \leq K \in \mathbb{R}, \forall y \in \overline{\mathcal{D}_{t}}$ and $t \in[0, T]$.

Strict concavity of the value function (implied by Condition A) implies that $V_{z}(z, t)$ is a decreasing function of $z$ and it is possible to define:

$$
z(y, t)=\inf \left\{z: z>0, \quad V_{z}(z, t)<y\right\}
$$

and the relation $z(y, t): \mathcal{D}_{t} \rightarrow\{z>0\}$ is one to one. As a consequence the Fenchel Legendre transform can be inverted and the following parametric representation of $V(z, t)$ holds:

$$
\begin{aligned}
V(z, t) & =\widetilde{V}(z(y, t), t)+z(y, t) y \quad y \in \mathcal{D}_{t} \\
z & =z(y, t)
\end{aligned}
$$

Hence the determination of the convex dual function $\widetilde{V}(y, t)$ for $y \in \mathcal{D}_{t}$ uniquely identifies $V(z, t)$ for $z>0$.

In addition, in the domain $\mathcal{D}_{t}$, differentiation of the convex duality relation implies that the following relations between the derivatives of $V(z, t)$ and $\widetilde{V}(y, t)$ hold:

$$
y=V_{z}(z, t), \quad \widetilde{V}_{y}(y, t)=-z, \quad V_{z z}(z, t)=-\frac{1}{\widetilde{V}_{y y}(y, t)},
$$

and the last equation is obtained from the first two relations as follows:

$$
V_{z z}(z, t)=\frac{d V_{z}(z, t)}{d z}=-\frac{d y}{d \widetilde{V}_{y}(y, t)}=-\frac{1}{\widetilde{V}_{y y}(y, t)} .
$$

Direct substitution of these relations in the HJB equation (11) for $V(z, t)$ implies that $\widetilde{V}(y, t)$ in $y \in \mathcal{D}_{t}$ is determined by the (convex dual) HJB differential equation:

$$
\begin{gathered}
H J B[\widetilde{V}(y, t)] \triangleq \widetilde{V}_{t}(y, t)+K_{1} \widetilde{V}(y, t)-\left(K_{1}+K_{2}\right) y \widetilde{V}_{y}(y, t) \\
+K_{3} y^{2} \widetilde{V}_{y y}(y, t)-K_{4} \frac{\left(\widetilde{V}_{y}(y, t)\right)^{2}}{\widetilde{V}_{y y}(y, t)}+F(y)=0
\end{gathered}
$$


with terminal boundary condition:

$$
\lim _{t \rightarrow T^{-}} \widetilde{V}(y, t)=\left(y-\beta^{1 / \gamma} \frac{y^{b}}{b}\right) \quad y \in \mathcal{D}_{T}=(0, \beta)
$$

A term by term derivation of eq.(14) with respect to $y$ implies the following equation for $z(y, t)=-\widetilde{V}_{y}(y, t)$ in $\mathcal{D}_{t}$ :

$$
\begin{aligned}
\Lambda[z(y, t)]: & =\frac{\partial}{\partial t} z(y, t)-\left[K_{2}+2 K_{4}\right] z(y, t)-\left(K_{1}+K_{2}-2 K_{3}\right) y z_{y}(y, t) \\
& +\frac{1}{2}\left(2 K_{3}+2 K_{4} \frac{z(y, t)^{2}}{y^{2} z_{y}(y, t)^{2}}\right) y^{2} z_{y y}(y, t)-y^{-1 / \gamma}+\delta=0
\end{aligned}
$$

\subsection{The dual value function in $\mathcal{D}_{t} \cup \mathcal{D}_{t}^{c}$ and the condi- tions at the boundary $y_{B}(t)$}

Consider now the domain $\mathcal{D}^{c}$. In this region the non-increasing property of $V_{z}(z, t)$ implies that the constraint $z \geq 0$ is binding. Recalling the relation $z=-\widetilde{V}_{y}(y, t)$, it is immediate to verify that $\mathcal{D}_{t}$ corresponds to the region where $-\widetilde{V}_{y}(y, t)>0$ and $H J B[\widetilde{V}(y, t)]=0$. $\mathcal{D}_{t}^{c}$ is a subset of the region identified by the condition, $z(y, t)=0$. Extending the relation $z=-\widetilde{V}_{y}(y, t)$ to $\mathcal{D}_{t}^{c}$ and following a standard construction, the definition of the reduced dual value function $\widetilde{V}(y, t)$ can be extended to the complete domain $\mathcal{D}_{t} \cup \mathcal{D}_{t}^{c}$ considering the variational inequality:

$$
\min \left\{H J B[\widetilde{V}(y, t)],-\widetilde{V}_{y}(y, t)\right\}=0
$$

and $\mathcal{D}_{t}^{c}$ is characterized by a constant dual value function, in fact $z(y, t)=$ $-\widetilde{V}_{y}(y, t)=0$ while $H J B[\widetilde{V}(y, t)]>0$. Repeating the same argument, the optimal function $z(y, t)$ solves:

$$
\min \{\Lambda[z(y, t)], z(y, t)\}=0
$$

This equation implies that at optimality the optimal liquid wealth plus the total consumption up to time $t$ evolves like a martingale. To complete the identification of the dual value function which produces the solution to the original optimization problem and state the corresponding Verification theorem, the variational equation (16) has to be completed with a set of lateral boundary conditions to be verified by $\widetilde{V}(y, t)$ in the limit $y \rightarrow y_{B}(t)$. In the following we introduce the proper set of lateral conditions. First, the 
regularity of $\widetilde{V}(y, t)$ postulated in Condition $\mathbf{A}$ and the definition of $y_{B}(t)$ require

$$
\lim _{y \rightarrow y_{B}(t)^{-} \in \mathcal{D}_{t}}-\widetilde{V}_{y}(y, t)=0
$$

In Regime $I$ the boundary point $y_{B}(t)$ is a point of separation of $\mathcal{D}_{t}$ from $\mathcal{D}_{t}^{c}$, i.e. $\overline{\mathcal{D}_{t}} \cap \overline{\mathcal{D}_{t}^{c}}=\left\{y_{B}(t)\right\}$, hence continuity of the dual value function imposes that the left limit of $\widetilde{V}(y, t)$ for $y \rightarrow y_{B}(t)^{-} \in \mathcal{D}_{t}$ and the right limit of $\widetilde{V}(y, t)$ for $y \rightarrow y_{B}(t)^{+} \in \mathcal{D}_{t}^{c}$ are equal:

$$
\lim _{y \rightarrow y_{B}(t)^{-}} \tilde{V}(y, t)=\lim _{y \rightarrow y_{B}(t)^{+}} \tilde{V}(y, t) \quad \forall t \in\left[0, \tau^{*}\right]
$$

In Regime $I I \mathcal{D}_{t}^{c}$ is disconnected from $\mathcal{D}_{t}$, thus the regions $\mathcal{D}_{t}$ and $\mathcal{D}_{t}^{c}$ are disjoint i.e. $\overline{\mathcal{D}_{t}} \cap \overline{\mathcal{D}_{t}^{c}}=\varnothing$, hence condition (19) is not necessary.

Last but not least, the admissibility conditions impose that at zero wealth the agent can invest a fraction of the liquid dividend only in the riskless asset, or equivalently, that he $\backslash$ she sets the subjective price of risk is set at zero. The condition that the price of insurable risk is zero determines the additional requirement:

$$
\lim _{y \rightarrow y_{B}(t)^{-} \in \mathcal{D}_{t}} \widetilde{V}_{y y}(y, t)=\lim _{y \rightarrow y_{B}(t)^{-} \in \mathcal{D}_{t}}-z_{y}(y, t)=0
$$

while the condition that the price of the uninsurable risk component is vanishing implies the limiting condition for the nonlinear term in eq.(14):

$$
\lim _{y \rightarrow y_{B}(t)^{-} \in \mathcal{D}_{t}} \frac{\widetilde{V}_{y}(y, t)}{y \widetilde{V}_{y y}(y, t)}=0
$$

The financial implications of these conditions will be clarified by the analysis of the (singular) optimal consumption and allocation policies at zero liquid wealth.

\subsection{A series expansion solution of the dual value func- tion $\widetilde{V}(y, t)$}

The boundary value problem formed by eq. (16)) jointly with the conditions (18), (19), (20) and (21) admits a formal series expansion solution of the form:

$$
\widetilde{V}(y, t)=y^{1-\frac{1}{\gamma}}\left\{B_{0}(t)+\sum_{n=1}^{\infty} y^{\frac{n}{\gamma}} B_{n}(t)\right\}
$$

It is easy to show that the first term in the expansion corresponds exactly to the Merton solution in complete markets. The series expansion solution 
is thus specified by the computation of the coefficients $\left\{B_{n}(t)\right\}_{n \in \mathbb{N}}$. They can be computed recursively inserting the formal expansion (22) in the HJB equation (14) and requiring that the equivalent equation (for $\left.\widetilde{V}_{y y}(y, t) \neq 0\right)$ :

$$
\begin{aligned}
0= & {\left[\widetilde{V}_{t}(y, t)+K_{1} \widetilde{V}(y, t)-\left(K_{2}+K_{1}\right) y \widetilde{V}_{y}(y, t)+K_{3} y^{2} \widetilde{V}_{y y}(y, t)\right] y^{2} \widetilde{V}_{y y}(y, t) } \\
& +F(y) y^{2} \widetilde{V}_{y y}(y, t)-K_{4} y^{2}\left[\widetilde{V}_{y}(y, t)\right]^{2}
\end{aligned}
$$

is valid. In the Appendix we show the result that after the insertion of the expression (22) the HJB equation becomes equivalent to a system of linear ODE with respect to time, which determine the coefficients $\left\{B_{n}(t)\right\}_{n \in \mathbb{N}}$. The numerical computation of the coefficients shows that the coefficients have alternate signs and a fast decay to zero. The terminal boundary conditions impose additional constraints on the coefficients $\left\{B_{n}(t)\right\}_{n \in \mathbb{N}}$ :

$$
\begin{aligned}
& B_{0}(T)=-\frac{\beta^{1 / \gamma}}{b}, \\
& B_{1}(T)=+1, \quad n \geq 2 . \\
& B_{n}(T)=0 \quad n .
\end{aligned}
$$

Note that the series expansion guessed in eq.(22) could provide only a local approximation to the dual value function $\widetilde{V}(y, t)$ in the limit of $y \rightarrow 0$. On the other hand the optimal solution is selected by the lateral boundary conditions which require the convergence of the series expansion for $y \rightarrow y_{B}(t)$.

Hence further information on the convergence of the series expansion is required. The analysis of the convergence properties turns to be easier for the derived series which determines $\widetilde{V}_{y}(y, t)$. Recall that within the domain of convergence of eq.(22) it is possible to exchange the derivative operator with the summation and the expansion for $\widetilde{V}_{y}(y, t)$ is given by:

$$
\widetilde{V}_{y}(y, t)=y^{-\frac{1}{\gamma}}\left\{B_{0}(t)+\sum_{n=1}^{\infty} y^{\frac{n}{\gamma}}\left(1-\frac{1}{\gamma}+\frac{n}{\gamma}\right) B_{n}(t)\right\} .
$$

The mathematical and economic interpretation of this expansion is clarified by making use of the relations $z(y, t)=-\widetilde{V}_{y}(y, t)$ and $c \triangleq c_{*}(z, 1, t)=$ $y^{-1 / \gamma}$ which hold at the optimum. Then it is straightforward to state:

Proposition 1 The trial solution (22) is an asymptotic solution to the HJB (14) for $y \rightarrow 0$ if and only if the function $z(c, t)$ which determines scaled liquid wealth $z$ as a function of scaled optimal consumption $c \triangleq c_{*}(z, 1, t)$ has the following asymptotic expansion:

$$
z(c, t) \stackrel{c \rightarrow+\infty}{\simeq}-\left\{B_{0}(t) c+B_{1}(t)+\sum_{n=2}^{\infty}\left(1-\frac{1}{\gamma}+\frac{n}{\gamma}\right) B_{n}(t) c^{-n}\right\}
$$


Assume that $z(c, t)$ is an increasing analytic function (i.e. with a converging Taylor expansion) in $c \in\left(c_{B}(t),+\infty\right)$ where $c_{B}(t) \triangleq y_{B}(t)^{-1 / \gamma}$ which satisfies the (interpolation) conditions:

$$
\lim _{c \rightarrow c_{B}^{-}(t)} z(c, t)=0 \quad \lim _{c \rightarrow c_{B}^{-}(t)} z_{c}(c, t)=0 \quad \lim _{c \rightarrow c_{B}^{-}(t)} \frac{c z_{c}(c, t)}{z(c, t)}=0
$$

then the dual reduced value function $\widetilde{V}(y, t)$ solving the optimization problem is determined by:

$$
\widetilde{V}(y, t)=y^{1-\frac{1}{\gamma}} G\left(y^{-\frac{1}{\gamma}}, t\right)
$$

where

$$
G\left(y^{-\frac{1}{\gamma}}, t\right)=-\frac{1}{y} \int_{0}^{y} z\left(x^{-\frac{1}{\gamma}}, t\right) d x
$$

In Theorem 5 it will be proved that the analytic property of $z(c, t)$ is implied by the optimal stochastic dynamics for consumption. It is worth noticing that Calin, Chen, Cosimano and Himonas (2005) use an analytic series expansion to solve equilibrium asset pricing models in complete markets. Our approach can be considered as an extension of their method to the (partial equilibrium) incomplete markets case.

\subsection{The main theorem.}

The application of the traditional dynamic programming approach and the above considerations lead us to state the following

Theorem 2 Assume Condition $\boldsymbol{A}$ and the following restrictions on parameters: $\delta>0,|\rho|\left\langle 1, \delta^{-\gamma}>\beta,\left(K_{1}+K_{2}-2 K_{3}\right)>0\right.$. Then the following assertions are true:

- The boundary curve $\left(t, y_{B}(t)\right), 0 \leq t \leq T$, is given by:

$$
y_{B}(t)=\beta \exp \left(-\left(K_{1}+K_{2}-2 K_{3}\right)(T-t)\right)
$$

- Let $V_{>}(z, t):(0,+\infty) \times[0, T] \rightarrow \mathbb{R}$ be defined by:

$$
V_{>}(z, t)=\inf _{y \in \mathcal{D}_{t}=\left(0, y_{B}(t)\right)}\{\widetilde{V}(y, t)+z y\}
$$

where $\widetilde{V}(y, t), y \in\left(0, y_{B}(t)\right)$, is the solution (27) to the boundary value problem formed by eq. (16) jointly with the conditions (18), (19), (20) 
and (21) and $\widetilde{V}_{B}(t)$ be the solution to the ODE:

$$
\begin{aligned}
\frac{d}{d t} \widetilde{V}^{B}(t) & =-K_{1} \widetilde{V}^{B}(t)+F_{0}\left(y_{B}(t)\right), \\
\lim _{t \rightarrow T^{-}} \widetilde{V}^{B}(t) & =\frac{\beta}{1-\gamma},
\end{aligned}
$$

then it is possible to define a concave function

$$
V(z, t)=\left\{\begin{array}{cc}
V_{\gtrsim}(z, t) & z>0 \\
\widetilde{V}_{B}(t) & z=0
\end{array}\right.
$$

and the function

$$
\begin{aligned}
V(l, h, t) & =h^{1-\gamma} V(z, t) \\
z & =l / h
\end{aligned}
$$

is the value function (5) which solves (6) in $(l, h, t) \in[0,+\infty) \times$ $(0,+\infty) \times[0, T]$.

- Let $\tau^{*}$ be defined as in eq.(46). Then $\tau^{*}$ separates two different regimes depending on the time to liquidation of the illiquid asset:

$$
\begin{array}{cll}
\text { Regime } I & y_{B}(t)^{-1 / \gamma} \geq \delta & 0 \leq t<\tau^{*} \\
\text { Regime } I I & y_{B}(t)^{-1 / \gamma}<\delta & \tau^{*} \leq t<T
\end{array}
$$

In Regime $I$ the reduced value function $V(z, t)$ admits a continuous extension to the domain: $[0,+\infty) \times[0, T]$. In Regime $I I$ the reduced value function $V(z, t)$ is discontinuous in the limit $z \rightarrow 0^{+}$for $\tau^{*}<$ $t<T$ :

$$
\lim _{z \rightarrow 0^{+}} V(z, t) \neq V(0, t)=\widetilde{V}^{B}(t)
$$

Proof The theorem is proved in the Appendix. End Proof

The value function is concave but possibly discontinuous in the domain $(t, z) \in(0, T) \times[0,+\infty)$. Lack of regularity of the solution complicates the application of the dynamic programming verification approach to the primal problem. For this reason the proof makes use of the dual verification approach discussed in Mnif and Pham (2001) which extends to the incomplete market case the duality method explored by He and Pagès (1991) and El Karoui and Jeanblanc (1997). 


\section{Stochastic evolution for the dual variable $y_{t}$}

The feedback formula (7) implies that $y$ has the economic interpretation of a (scaled) shadow price for the individual's optimal consumption plans. In the approach of He and Pagès (1991) and El Karoui and Jeanblanc (1997), the optimal stochastic dynamics for the dual variable $y$ is determined as a solution to a dual stochastic minimization problem. In our derivation the singular diffusion process for $y_{t}$ can be reconstructed from eq.s $(16,17)$ and by the set of lateral boundary conditions. This is stated in the next:

Proposition 3 Let $L^{y_{B}(t)}\left(y_{t}\right)$ the local time of the process $y_{t}$ at the boundary $y_{B}(t)$. Then optimal stochastic dynamics for the shadow price of consumption $y_{t} \in\left(0, y_{B}(t)\right)$ for $0 \leq t \leq \tau_{B}^{*}(\omega)$ is given by:

$$
\begin{aligned}
\frac{d y_{t}}{y_{t}}= & -\frac{1}{2} \frac{d L^{y_{B}(t)}\left(y_{t}\right)}{y_{B}(t)}+ \\
+ & {\left[-\left(K_{1}+K_{2}-2 K_{3}\right) d t+\sqrt{2 K_{3}} d W_{t}^{1}+\sqrt{2 K_{4} \widetilde{R}\left(y_{t}, t\right)^{2}} d W_{t}^{2}\right] \mathbb{I}_{\left\{y_{t}<y_{B}(t)\right\}} }
\end{aligned}
$$

The initial condition $y_{0}$ corresponding to $z_{0}=l / h$ is uniquely determined by the equation:

$$
z_{0}=-\widetilde{V}_{y}\left(y_{0}, 0\right)
$$

By construction $y_{t}$ is reflected at $y_{B}(t)$ at any boundary hitting time:

$$
\tau_{t}^{R}(\omega)=\inf \left\{\tau \geq t: \quad t<\tau<\tau^{*}, \quad \lim _{\tau^{\prime} \rightarrow \tau^{-}} y_{\tau^{\prime}}(\omega)=y_{B}(\tau)\right\}
$$

while at the killing time:

$$
\tau^{B}(\omega)=\inf \left\{\tau \geq 0: \quad \tau^{*}<\tau \leq T, \quad \lim _{\tau^{\prime} \rightarrow \tau^{-}} y_{\tau^{\prime}}(\omega)=y_{B}(\tau)\right\}
$$

(with the convention that inf $\varnothing=T$ ) the process collapses to the state $y_{t}=$ $\delta^{-\gamma}$ where it remains until liquidation.

Proof See the Appendix. End Proof

Straightforward application of Ito lemma to the relations $c=y^{-1 / \gamma}$ and $z(y, t)=-\widetilde{V}_{y}(y, t)$ determines the optimal evolution of consumption $c_{t}$ and scaled liquid wealth $z_{t}$. 


\subsection{The (singular) consumption and allocation policies at zero liquid wealth}

Having completed the analysis of the boundary behavior for the dual state process $y_{t}$, it is now possible to provide a complete description of the optimal control policies at zero liquid wealth which can be summarized as follows:

- Regime $I: 0<t \leq \tau^{*}$ The agents' savings produce a positive inflow of wealth that determine the instantaneous exit from the illiquid state $l=0$. When the zero wealth boundary is hit, the agent can consume only out of the dividend rate. Since the constraint $y>\delta^{-\gamma}$ is not binding, the agent's optimal consumption level is strictly smaller than the dividend rate:

$$
c_{*}^{B}(t)<\delta h
$$

hence the agent saves a fraction of the dividend rate which is invested in the riskless asset and instantaneously exits from the zero liquid wealth state.

- Regime $I I: \tau^{*} \leq t \leq T$ If the zero liquid wealth boundary is reached after $\tau^{*}$, the proximity of the liquidation time $T$ raises the agent's willingness to consume beyond the dividend rate level $\delta h$. Since borrowing is not permitted, the maximum amount is limited to the available liquid wealth which equals the dividend rate. This implies that the no-borrowing constraint binds and the optimal consumption level must be set equal to the dividend rate:

$$
c_{*}^{B}(t)=\delta h
$$

until final liquidation. The agent in this state does not save thus the allocation to liquid risky and riskless assets is 0 .

\subsection{The regularity of the (scaled) wealth-consumption function $z(c, t)$}

In the original Merton model the lognormal property of optimal consumption implies a linear wealth-consumption function. A similar argument can be used to characterize the regularity of the (scaled) wealth consumption function in the illiquid market framework. The next theorem states the following important extension of the complete market setup: first, the optimal scaled consumption evolution is in the class of the time changed Bessel processes, a class of processes which includes also lognormal ones. Second, the time changed Bessel property for optimal consumption dynamics implies that 
the optimal (scaled) wealth-consumption function $z(c, t)$ is analytic and is monotonic increasing. These two regularity properties, which are necessary in order to prove Prop.1 and Th.2, are proved by showing that the inverse of $z(c, t)$ belongs to a special class of functions which are defined in the following:

Definition 4 (C. Loewner 1964) A function $f:(0,+\infty)$ which is (real) analytic in $(0,+\infty)$ and can be analytically continued to a one-to-one map of the upper half complex plane $\mathbb{H}$ into itself is said to be a "conformal transformation". Each conformal function $f$ is a matrix monotone function of arbitrary order ${ }^{\mathrm{e}}$ and is generated by the composition of infinitesimal transformations with the same property.

To draw an analogy with familiar models, the class of conformal transformations in this framework plays the role which exponential affine functions play for traditional affine factor models. As the family of exponential affine functions is mapped in itself by the affine factor dynamics, in the same way the (time changed) Bessel dynamics maps the class of inverse conformal transformations in itself. This connection has been recently discovered in Bernard and Bauer $(2002,2003)$ in connection with the recent developments in the analysis of Schramm Loewner evolutions, see Schramm Lawler Werner (2003). Then we can state:

Theorem 5 Consider the following rescaling of variables:

$$
\begin{aligned}
\widetilde{\delta}_{1}(T-t) & =\delta \exp \left[\frac{\left(K_{1}+K_{2}-2 K_{3}\right)+\gamma\left(K_{2}+2 K_{4}\right)}{\gamma}(T-t)\right] \\
\widetilde{c}_{t} & =c_{t} \exp \left[\frac{\left(K_{1}+K_{2}-2 K_{3}\right)}{\gamma}(T-t)\right] \\
\widetilde{z}(\widetilde{c}, T-t) & =z(c, t) \exp \left[\left(K_{2}+2 K_{4}\right)(T-t)\right]
\end{aligned}
$$

then:

- Scaled consumption $\widetilde{c}$ evolves like a time changed Bessel process restricted to the state space $\widetilde{c} \in[1,+\infty)$ and reflected at the boundary

${ }^{\mathrm{e}} \mathrm{A}$ function $f$ is said matrix monotone of order $n$ in $(0,+\infty)$ if for any value $0<x_{1}<$ $y_{1}<\ldots<x_{m}<y_{m}, m \leq n$, the determinant of the matrix $M^{(m)}=\left[m_{i, j}\right]_{i, j=1, . . m}$ with elements

$$
m_{i, j}=\left|\frac{f\left(x_{i}\right)-f\left(y_{j}\right)}{x_{i}-y_{j}}\right|_{i, j=1}^{m}
$$

is positive, $\operatorname{det} M^{(m)} \geq 0$, for a discussion of their properties see Donoghue (1974) 
point for for $t<\tau_{B}(\omega), \widetilde{c}_{B}=1$ :

$$
\begin{aligned}
d \widetilde{c}_{\varphi_{t}} & =\frac{1}{2}(1+\gamma) \frac{d \varphi_{t}}{\widetilde{c}_{\varphi_{t}}}+d \widetilde{B}_{\varphi_{t}}+\frac{1}{2} d L_{\varphi_{t}}^{1}(\widetilde{c}) \\
\varphi_{t} & =2 \int_{0}^{t} \nu\left(\widetilde{c}_{s}, T-s\right) d s \quad t \leq \tau^{B}(\omega) \\
\nu(\widetilde{c}, T-s) & =2 \frac{\widetilde{c}^{2}}{\gamma^{2}}\left[K_{3}+K_{4}\left(\frac{\widetilde{z}(\widetilde{c}, T-s)}{\widetilde{c} \widetilde{z}_{\widetilde{c}}(\widetilde{c}, T-s)}\right)^{2}\right]
\end{aligned}
$$

- Assume $K_{3} \neq 0$, then the function $\widetilde{z}(\widetilde{c}, \tau)$ is (the inverse of) a conformal transformation, thus it is analytic and monotonic increasing and by Eq.(33) determines a unique real analytic increasing function $z(c, t)$.

- The analytic increasing function $z(c, t)$ determines a unique convex dual value function $\widetilde{V}(y, t)$ in $y \in\left(0, y_{B}(t)\right)$ by Proposition 1 eq. (27) which satisfies all the assumptions in Condition $\boldsymbol{A}$. Hence Theorem 2 can be applied and the solution to the original stochastic optimization problem is uniquely determined.

Proof The Theorem is proved in the Appendix. End Proof

This regularity result is extremely useful to simplify the analysis of optimal allocation and consumption choice in incomplete markets. In fact it makes available a broad spectrum of numerical techniques like interpolation and rational approximation schemes for analytic and univalent functions. A general introduction to these applied numerical methods is given in Henrici (1991).

The solution approach presented in this paper provides a constructive scheme to produce the numerical evaluation of the solution. In fact the computation of the solution is reduced to the computation of the analytic function $z(c, t)$ as defined by a set of interpolation conditions like (25), (26). Applied numerical analysis offers a variety of methods to reproduce an analytic function with an arbitrary accuracy using a polynomial or a rational function. In the numerical example we will practically exemplify the numerical determination of the solution. In particular it will be shown that the solution can be approximated by:

$$
\widetilde{V}_{y}^{(N)}(y, t)=y^{-1 / \gamma}\left(1-\frac{y^{1 / \gamma}}{y_{B}(t)^{1 / \gamma}}\right)^{2} P^{(N)}\left(y^{1 / \gamma}\left(1-\frac{y^{1 / \gamma}}{y_{B}(t)^{1 / \gamma}}\right), t\right)
$$

where $P^{(N)}$ is a polynomial function of degree $N-2$ such that $P^{(N)}(0) \neq 0$. The presence of a double root corresponding to $y^{1 / \gamma}=y_{B}(t)^{1 / \gamma}$ grants that 
this class of functions satisfies the required boundary conditions at $y=y_{B}(t)$. The exact expression of the polynomial function $P^{(N)}$ is uniquely determined by the condition that the expansion of $\widetilde{V}_{y}^{(N)}(y, t)$ as $y \rightarrow 0(c \rightarrow \infty)$ coincides with the $2 N$-th order truncation of (24). Specific numerical issues about the (fast) numerical convergence of this approximation are discussed in Appendix.

\section{Analysis of optimal consumption and allo- cation policies}

Having solved for the value function it is easy to proceed to determine the optimal consumption and asset allocation using feedback formulas. To provide for a better understanding of the solution obtained and to be able to relate our results to those existing in the current literature, we introduce a representation that allows us to get a better intuition of how the agent "values" his illiquid wealth and how his decisions are related to it.

\subsection{Marginal valuation of the illiquid asset}

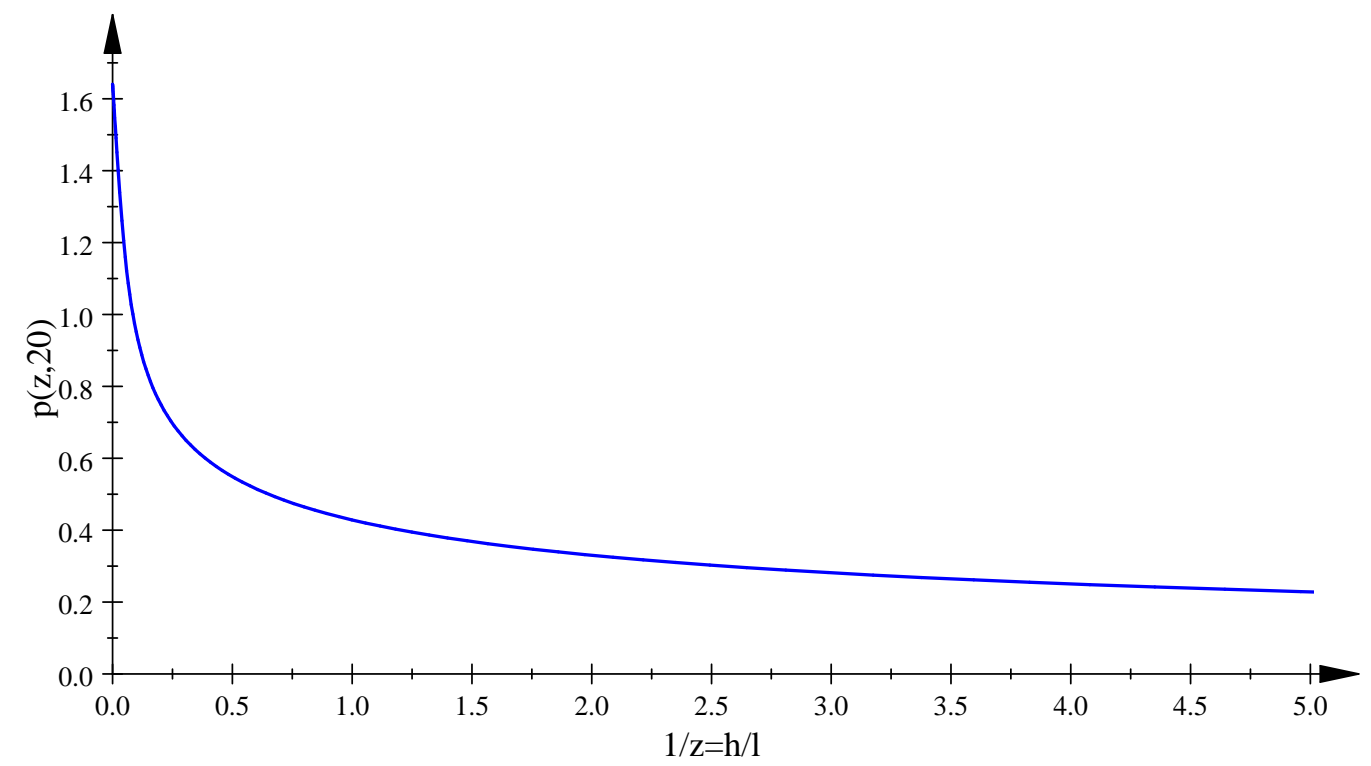

Figure 1: Marginal utility based price as a function of the ratio $1 / z=h / l$.

The marginal utility based value of the illiquid asset $p$ is defined as the liquid amount at which the investor would be willing to sell an infinitesimal 
amount $\varepsilon$ of the illiquid asset at time $t$ and is defined by:

$$
\begin{aligned}
V(l, h+\varepsilon, t) & \approx V(l+p \varepsilon, h, t)+O\left(\varepsilon^{2}\right) \\
V_{h}(l, h, t) & =p V_{l}(l, h, t) \\
p & :=\frac{V_{h}(l, h, t)}{V_{l}(l, h, t)} .
\end{aligned}
$$

The homogeneous representation of the value function, eq.(10), jointly with eqs.(66) in the Appendix show that $p$ is a function of the state variable $z=l / h$ and its expression in terms of the reduced value function $V(z, t)$ is given by:

$$
p(z, t)=(1-\gamma) \frac{V(z, t)}{V_{z}(z, t)}-z .
$$

In the case that the illiquid asset is private equity, the marginal utility based value of the illiquid asset $p(z, t)$ corresponds to the minimum price the agent would be willing to receive in order to sell an infinitesimal amount of the private equity.

For the purpose of comparing our analysis with the existing literature, in particular with Merton (1971) it is useful to define:

$$
w(l, h, t)=l+p(z, t) h=h(z+p(z, t))
$$

$w(l, h, t)$ is the equivalent total liquid wealth if the agent values his illiquid holding at the marginal value $p(z, t)$. Following Koo (1998) we refer to $w(l, h, t)$ as implicit total wealth. Using eqs.(66), it is immediate to verify that:

Proposition $6 V(l, h, t)$ is concave in the variables $(l, h)$ if and only if $V(z, t)$ is concave and $p_{z}(z, t)>0$ for $z>0$.

Since $\lim _{z \rightarrow 0^{+}} p(z, t)=0$, the increasing property of $p(z, t)$ implies also the positivity of the marginal price. Thus a direct numerical verification of concavity can be produced using the feed-back formula:

$$
p(z(y, t), t)=\sum_{n=0}^{+\infty} n B_{n}(t) y^{\frac{1-n}{\gamma}} \quad y \in\left(0, y_{B}(t)\right)
$$

and checking the increasing property of $p_{z}(z, t)$.

To gain insight into the results obtained we illustrate our findings through a numerical example. Consider an investor with an horizon of 20 years, a coefficient of risk aversion of $\gamma=3$, and $\beta=1$ who is allocating funds to a liquid risky asset with an expected rate of return of $\alpha=0.08$ and 
volatility $\sigma=0.15$. In addition he holds an illiquid risky asset with a drift $\mu=0.08$ and volatility $\eta=0.20$. Even though the illiquid asset can not be traded, it pays a liquid dividend yield of $\delta=0.05$. Finally, there is a liquid riskless asset with a constant interest rate $r=0.03$. Observe that within this numerical example $\left(K_{1}+K_{2}-2 K_{3}\right)=0.093022$, thus $\tau^{*}$ solves the equation $T-\tau^{*}=96.613$. Since we analyze an investment which is liquidated over $T=20<96.613$ years hence the constraint is always binding for any positive time to liquidation. In the illustration we consider as liquidity state variable $h / l$, the proportion of illiquid wealth per unit of liquid wealth within the range of parameters where the nominal proportion of illiquid wealth varies from 0 to 5 times the liquid holdings of the agent. In Table I we report the parameters we used in the illustration.

Table I. Parameters of the illustrative example

\begin{tabular}{|l|l|l|l|l|l|l|l|l|l|l|}
\hline$T$ & $\alpha$ & $\sigma$ & $\mu$ & $\eta$ & $\rho$ & $\delta$ & $r$ & $\kappa$ & $\gamma$ & $\beta$ \\
\hline 20 & 0.08 & 0.15 & 0.08 & 0.2 & 0.2 & 0.05 & 0.03 & 0.05 & 3 & 1 \\
\hline
\end{tabular}

Figure 1 shows the marginal utility based value as a function of liquidity expressed as the proportion of illiquid wealth per unit of liquid wealth, $1 / z=h / l$, for the parameters given in Table I. When $h \rightarrow 0, p$ converges to a constant implying that the investor would be willing to sell at this price his total illiquid wealth, which at this point becomes an infinitesimal fraction of liquid one (in Section 5 we compute this price). Increasing $h / l, p$ decreases monotonically, hence the more illiquid the agent, the less valuable is an infinitesimal share of his illiquid wealth. When $h / l \rightarrow \infty, p$ converges to 0 .

\subsection{Optimal consumption over wealth ratio}

For purposes of comparison it is useful to define the consumption over implicit total wealth ratio as:

$$
q:=\frac{c^{*}(l, h, t)}{w(l, h, t)}
$$

where $c^{*}(l, h, t)=\left[V_{l}(l, h, t)\right]^{-1 / \gamma}$ is the expression for optimal consumption obtained maximizing eqs.(7). An easy verification shows that also $q$ is independent of $h$ and is a function of $z$ and $t$ only.

In the appendix we prove that the value function can be expressed in terms of $q(z, t)$ and $w(l, h, t)$ as:

$$
V(l, h, t)=q^{-\gamma}(z, t) \frac{w(l, h, t)^{1-\gamma}}{1-\gamma} .
$$




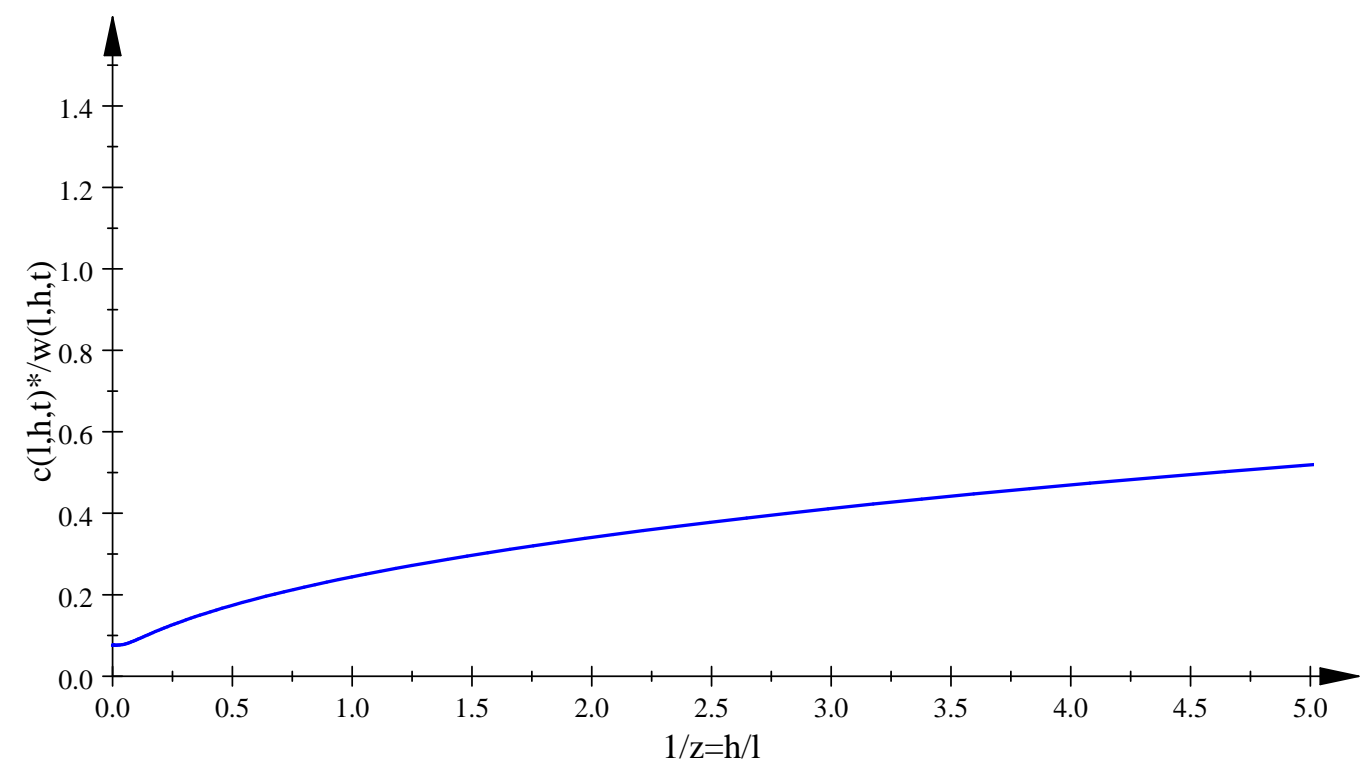

Figure 2: Consumption over implicit total wealth ratio as a function of $1 / z=$ $h / l$

This representation shows that for a fixed value of the state variable $z$ the value function assumes the standard Merton form, where $w(l, h, t)$ is now the implicit valuation of the agent total wealth. Similarly, a liquidity constrained investor will consume a fraction $q(z, t)$ of his implicit total wealth. In Figure 2 we show the value of $q$ as a function of illiquidity $1 / z=h / l$ for the parameters given in Table I. The limit value of $q(z, t)$ when $h \rightarrow 0$ is given by the optimal consumption over wealth ratio when all wealth is liquid (Merton 1971) and is given by:

$$
\begin{aligned}
q^{M e r t}(t) & =\left(m^{-1}\left(e^{m(T-t)}-1\right)+\beta^{1 / \gamma} e^{m(T-t)}\right) \\
m & =\frac{\kappa}{\gamma}-r\left(1-\frac{1}{\gamma}\right)-\frac{1}{2}\left(\frac{\alpha-r}{\sigma}\right)^{2}\left(\frac{1}{\gamma}-\frac{1}{\gamma^{2}}\right) .
\end{aligned}
$$

Notice that the value of $q(z, t)$ remains finite (although not constant) as the liquid wealth goes to zero, implying that the agent will consume a fraction of its implicit total wealth, even if he can consume only the liquid asset.

\subsection{Optimal allocation strategy}

The optimal allocation is obtained by performing the maximization in (8). Then, expressing the value function in terms of the reduced value function 


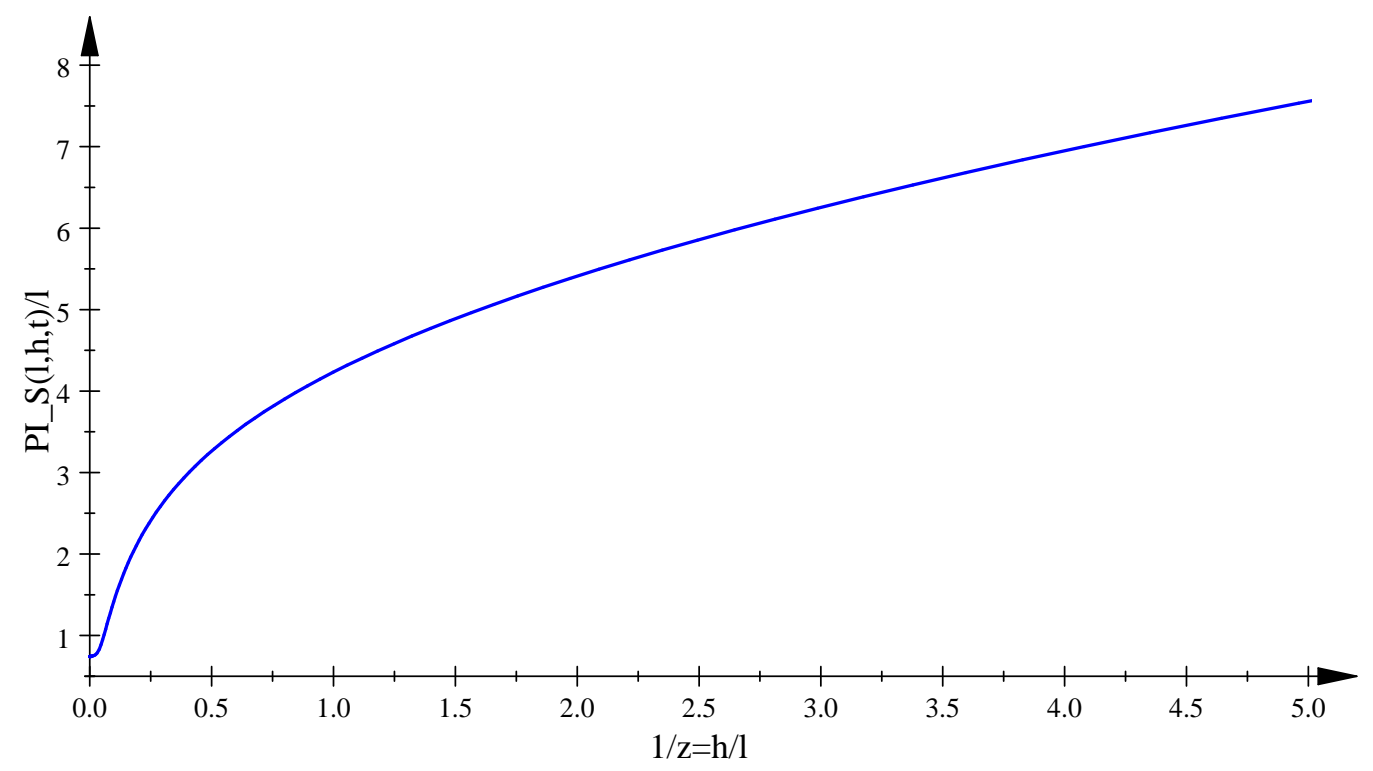

Figure 3: Fractional allocation to the risky liquid asset as a function of $1 / z=h / l$.

$V(z, t)$, the optimal allocation to the risky liquid asset can be written as:

$$
\pi^{S}=\left[\frac{(\alpha-r)}{\sigma^{2}}\left(\frac{1}{R(z, t)}\right)-\frac{\eta \rho}{\sigma}\left(\frac{\gamma}{R(z, t)}-1\right)\right] l,
$$

where $R(z, t)$ is defined as:

$$
R(z, t)=-\frac{z V_{z z}(z, t)}{V_{z}(z, t)} .
$$

Figure 3 shows the fraction of liquid wealth allocated to the liquid risky asset. When $h / l=1 / z \rightarrow 0, R(z, t)$ converges to the risk aversion coefficient $\gamma$, hence the first term in eq.(38) becomes Merton's (1971), while the second term, the intertemporal hedging component induced by the presence of the illiquid asset, disappears. When $h / l \rightarrow \infty, R(z, t) \rightarrow 0$ therefore when the agent has a very small amount of liquid wealth, he will invest a large fraction (the total amount in the limiting case of $z \rightarrow 0$ ) of his liquid holdings in the risky asset in order to diversify the risk induced by the illiquid asset and to maximize the probability to avoid the hitting of the zero wealth boundary. If the boundary is hit, the risky allocation is instantaneously liquidated. In Regime $I$ the optimal allocation policy for an agent $l=0$ would correspond to a pure riskless asset allocation. Figure 4 shows the allocation to the liquid risky asset per unit of illiquid wealth. The dollar allocation decreases and converges to 0 when $l \rightarrow 0$, while it diverges when $l \rightarrow \infty$. 


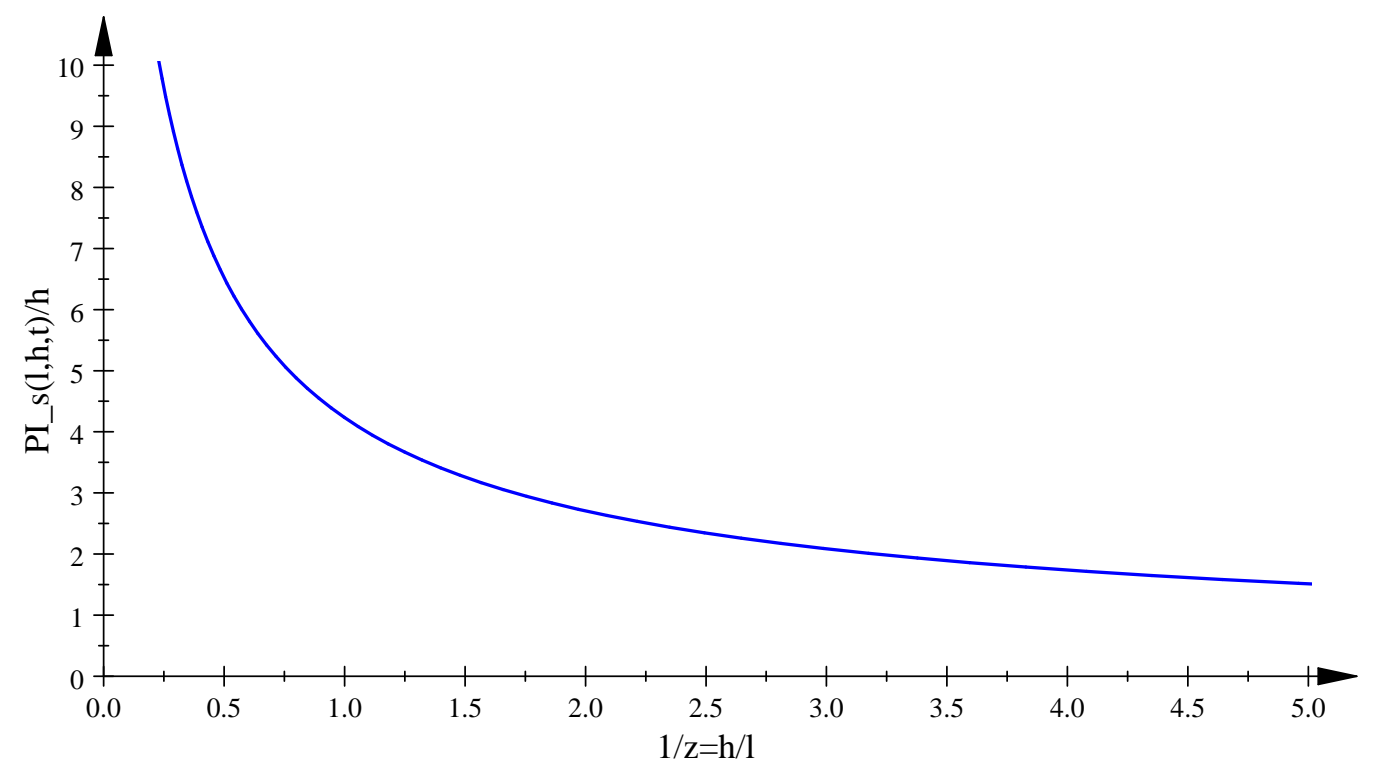

Figure 4: Allocation to the liquid risky asset per unit of illiquid wealth as a function of the $1 / z=h / l$.

\subsection{The individual valuation of illiquid assets and the price of uninsurable risk}

The dual approach that we take allowed us to compute the shadow prices for consumption or equivalently the marginal rates of substitution which the agent uses to price both market and the illiquid asset risk. In the region where the borrowing constraint is not binding, first order conditions impose that the marginal utility of consumption $\mathcal{U}_{c}\left(t, c^{*}, W_{T}^{\pi^{*}, c^{*}}\right)$ is proportional to the implicit Arrow Debreu prices which we denote by $\xi_{t}(\omega)$. Hence at the optimum:

$$
\mathcal{U}_{c}\left(t, c^{*}, W_{T}^{\pi^{*}, c^{*}}\right) \propto \xi_{t}
$$

The differential of the Stochastic Discount Factor which determines the evolution of the marginal utility of consumption can be easily obtained applying Ito Lemma to the combination: $H_{t}^{-\gamma} y_{t}$. Its variance is given by

$\operatorname{Var}\left[\frac{d \xi_{t}}{\xi_{t}}\right]=\operatorname{Var}\left[\frac{d\left(H_{t}^{-\gamma} y_{t}\right)}{H_{t}^{-\gamma} y_{t}}\right]=\left(\frac{(\alpha-r)}{\sigma}\right)^{2}+\left(\gamma-\widetilde{R}\left(y_{\tau}, \tau\right)\right)^{2} \eta^{2}\left(1-\rho^{2}\right)$

where $\widetilde{R}\left(y_{t}, t\right)$ is defined by the feedback formula:

$$
\widetilde{R}\left(y_{t}, t\right)=-\frac{\widetilde{V}_{y}\left(y_{t}, t\right)}{y \widetilde{V}_{y y}\left(y_{t}, t\right)} .
$$




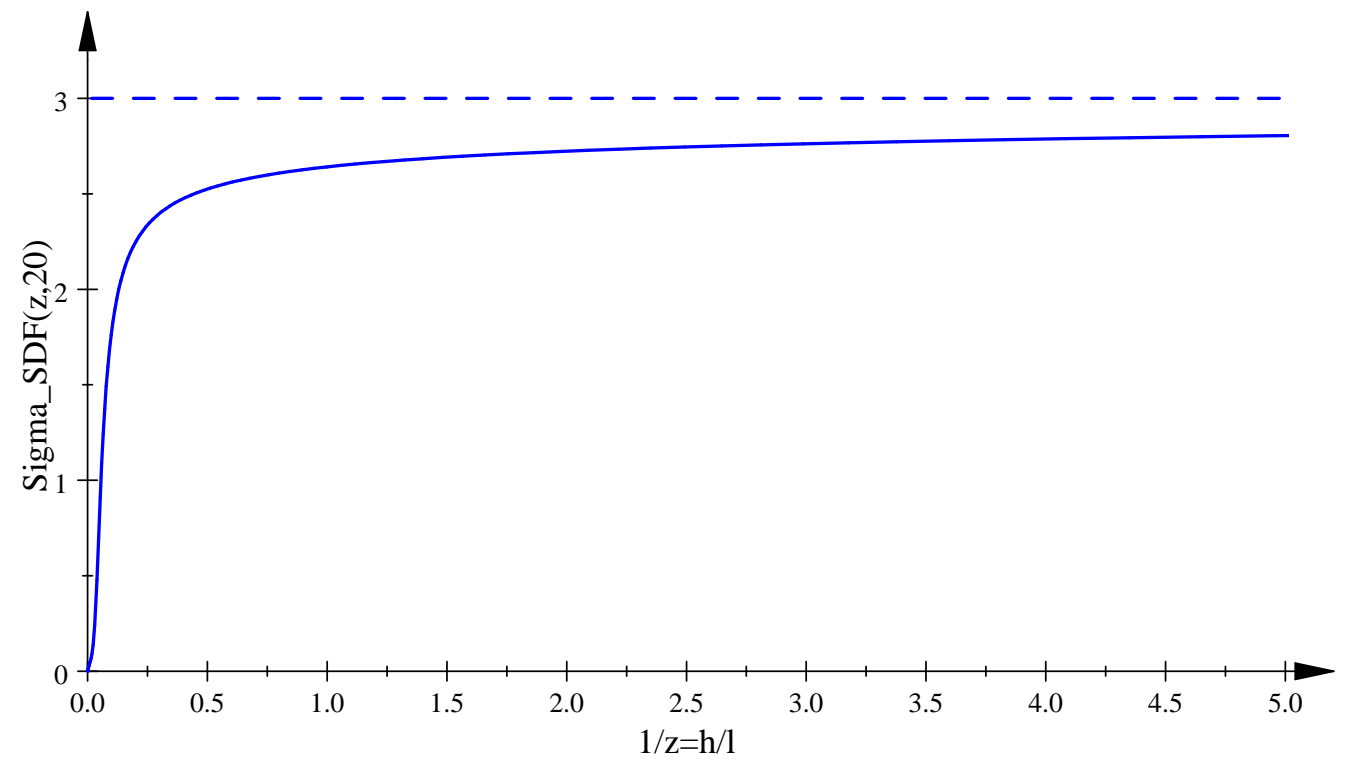

Figure 5: Risk adjustment over the non traded source of risk as a function of $1 / z=h / l$

It is immediate to separate two contributions $\sigma_{1}^{S D F} \triangleq(\alpha-r) / \sigma$ which corresponds to the market risk premium. The residual component:

$$
\sigma_{2}^{S D F} \triangleq\left(\gamma-\widetilde{R}\left(y_{t}, t\right)\right) \eta\left(1-\rho^{2}\right)^{1 / 2}
$$

depends on the source of risk which cannot be diversified by trading in the liquid market. Any choice of $\sigma_{2}^{S D F}$ will determine a different risk adjusted processes for cash flows whose evolution depend on the risk source $W_{t}^{2}$ and therefore to different valuations of the asset which are illiquid and correlated with the source $W_{t}^{2}$.

Observe that the expression obtained for $\sigma_{2}^{S D F}$ corresponds to the one discussed in Teplà (2000) and in He and Pearson (1991, Th.7, pg.287) in the context of the martingale duality approach to stochastic portfolio optimization in incomplete markets, then we can state:

Corollary 7 The optimal subjective price of uninsurable risk $\sigma_{2}^{S D F}$ is given by:

$$
\sigma_{2}^{S D F}(y, t)=(\gamma-\widetilde{R}(y, t)) \eta\left(1-\rho^{2}\right)^{1 / 2}
$$

Proof The theorem is proved in the Appendix. 
Notice that writing the expression for $\widetilde{R}(y, t)$ in terms of the primal state variable $z$ we get

$$
R(z, t)=\widetilde{R}(y(z, t), t)=-\frac{z V_{z z}(z, t)}{V_{z}(z, t)}
$$

which allows us to express the risk adjustment $\sigma_{2}^{S D F}$ as a function of the liquidity of the agent. In Figure 5 we plot the risk adjustment $\sigma_{2}^{S D F}$ per unit of unspanned volatility $\eta\left(1-\rho^{2}\right)^{1 / 2}$. In fact unspanned volatility $\eta\left(1-\rho^{2}\right)^{1 / 2}$ can be considered as a measure of market incompleteness, the larger it is, the more important the role of non-marketed risks. For a fixed level of unspanned volatility, we can immediately relate $\sigma_{2}^{S D F}$ to the risk aversion of the agent. When $h / l \rightarrow 0, R(z, t) \rightarrow \gamma$, thus $\sigma_{2}^{S D F} \rightarrow 0$ and the agent will value his illiquid holdings ignoring the non-traded risk. ${ }^{\mathrm{f}}$ In the limit when $\sigma_{2}^{S D F}=0$ only market risks are valued and the corresponding (marginal) value of the illiquid asset will be:

$$
\begin{aligned}
\widehat{h}_{t}^{\sigma_{2}^{S D F}=0} & =h_{t}\left[e^{-\nu(T-t)}+\delta \nu^{-1}\left(1-e^{-\nu(T-t)}\right)\right] \\
\nu & =-\left(\mu-\eta \rho \frac{(\alpha-r)}{\sigma}-\delta-r\right) .
\end{aligned}
$$

This point corresponds to the $h / l=0$ limit in Figure 1 .

When the agent liquid holdings become small and $h / l \rightarrow \infty$ then $R(z, t) \rightarrow 0$, thus $\sigma_{2}^{S D F} \rightarrow \gamma \eta\left(1-\rho^{2}\right)^{1 / 2}$. This upper bound for $\sigma_{2}^{S D F}$ is reached when the agent only owns the illiquid asset and corresponds to the risk adjustment on the unspanned source of risk required for the agent to optimally hold only the illiquid asset (see Gerber and Shiu 2000).

\section{Sensitivity analysis}

\subsection{Consumer choice}

Figure 6 and Figure 7 analyze the consumption choices of the agent as a function of his liquidity for different times to the final horizon. If no illiquid asset is held by the agent, $h / l=0$, and the standard Merton consumption over wealth ratio is recovered in both plots. Figure 6 , which considers the consumption per unit of liquid wealth as a function of $h / l$, shows that an increase in the proportion of the illiquid asset held by the agent will increase his consumption almost linearly.

\footnotetext{
${ }^{\mathrm{f}}$ Collin-Dufresne and Hugonnier (2006) show that this result holds in general semimartingale models.
} 


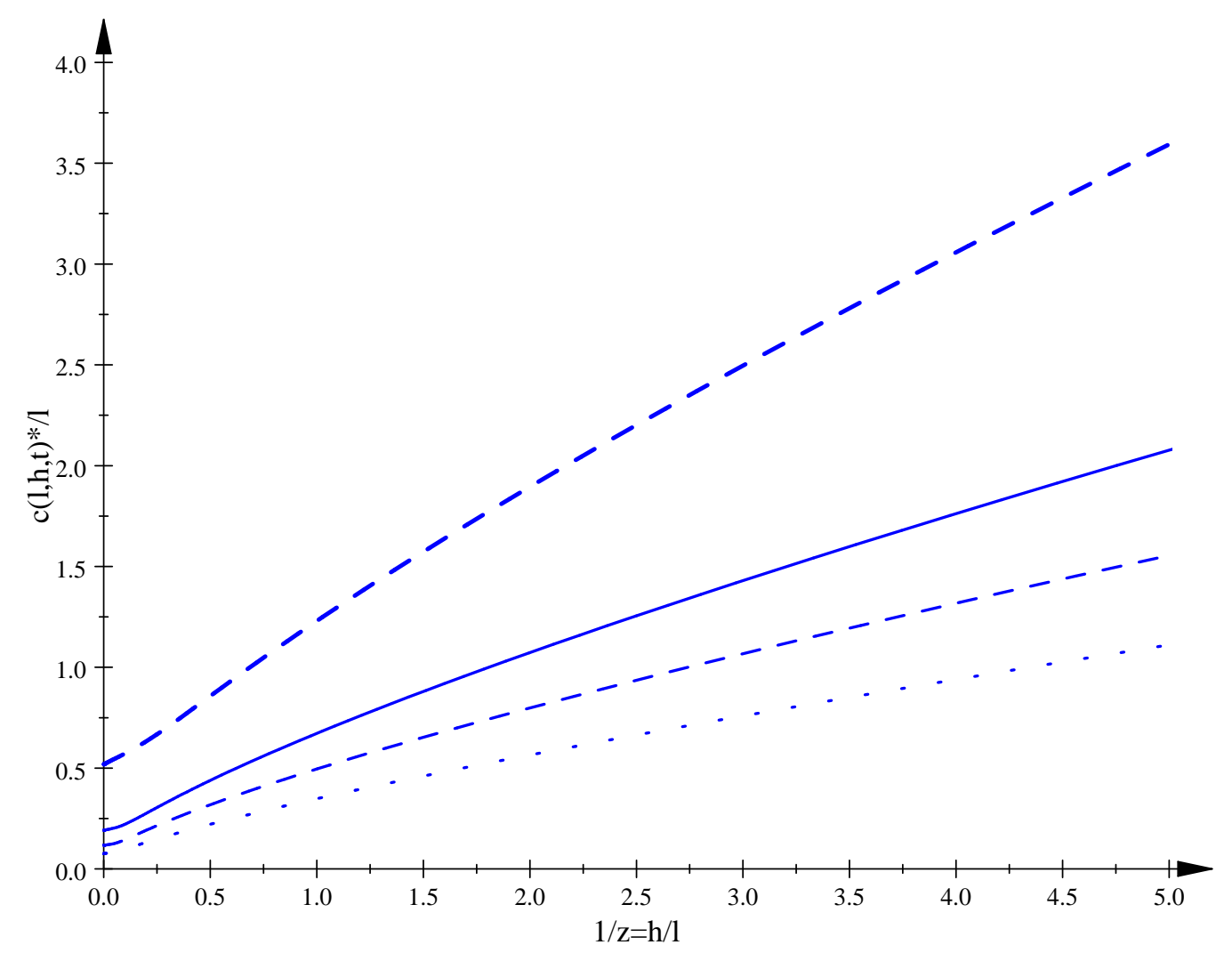

Figure 6: Consumption over liquid wealth ratio as a function of $h / l=1 / z$ for different times to the final horizon. $T-t=20$ Dotted, $T-t=10$ Dashed, $T-t=5$ Solid, $T-t=1$ Bold Dashed.

In Figure 7 we consider consumption per unit of total implicit wealth. It shows that consumption over implicit total wealth ratio has little variation as $h / l \rightarrow \infty$. Note that consumption increases as the final horizon is approached.

If we interpret the illiquid asset as human wealth (the discounted value of future labor income) then our findings can be related to the recent results in the consumption literature (see e.g. Wang (2006) and references therein). In particular, under the more realistic assumption of constant relative risk aversion preferences for the agent, we can analyze and provide analytical support for both the standard and generalized Friedman (1957) Permanent Income Hypotheses (PIH) as defined in Wang (2006). Within this framework, the state variable $z$ determines the fraction of financial wealth (cumulative savings) to human wealth. Assume that the agent values his (implicit) total wealth $w(l, h, t)$ according to formula (35). Then according to $\mathrm{PIH}$, the 


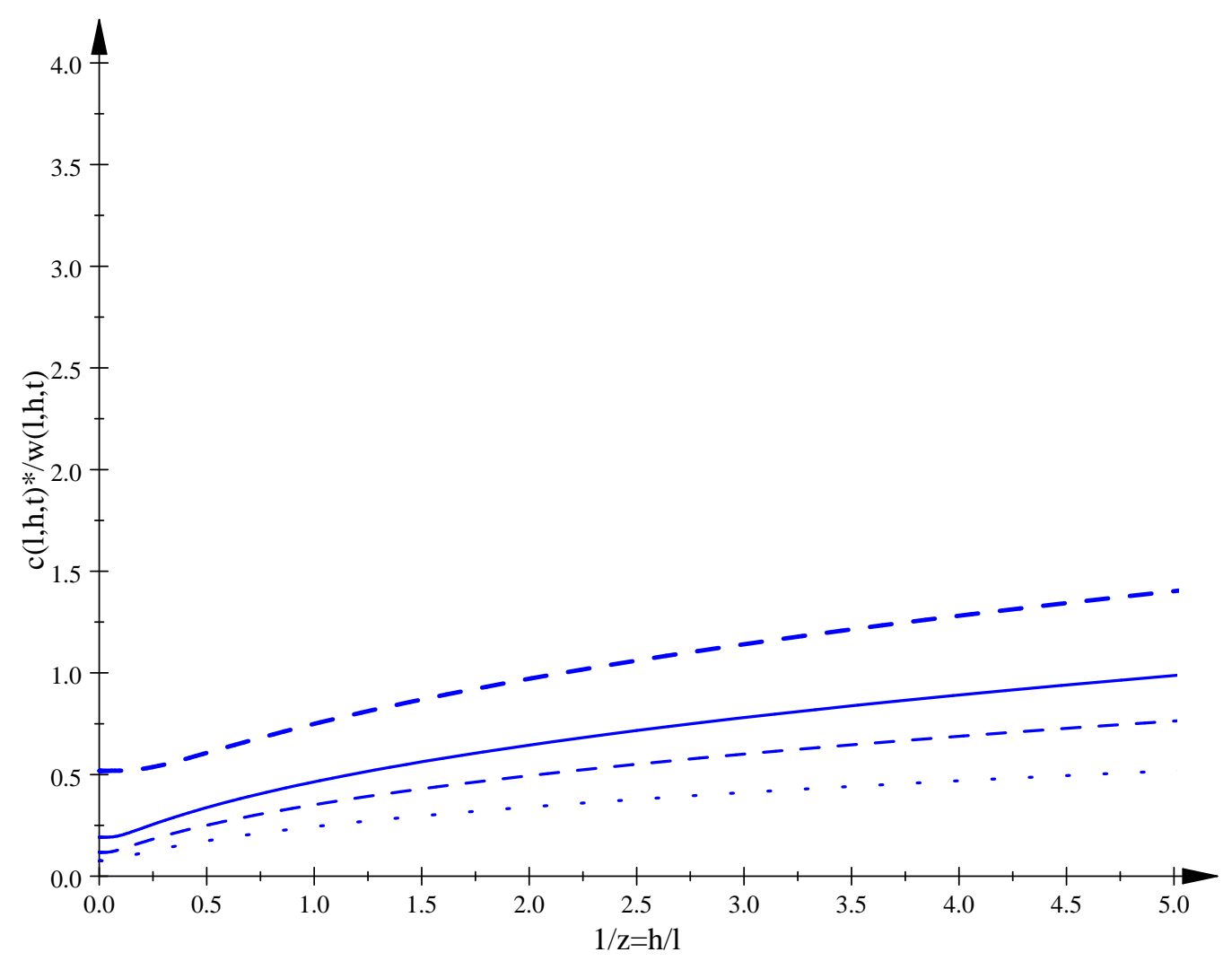

Figure 7: Consumption over implicit total wealth ratio as a function of $h / l=$ $1 / z$ for different times to the final horizon, $T-t=20$ Dotted, $T-t=10$ Dashed, $T-t=5$ Solid, $T-t=1$ Bold Dashed.

agent will consume a constant fraction of $w(l, h, t)$. Our model supports this prediction. In fact Figure 7 shows that the ratio of consumption to implicit total wealth has little variation with respect to the liquidity state of the agent for $h / l$ sufficiently large.

Moreover, the difference between the elasticity of consumption with respect to the liquid and to the illiquid wealth can be expressed in terms of the function $R(z, t)$ :

$$
\frac{l}{c^{*}(l, h, t)} \frac{\partial c^{*}(l, h, t)}{\partial l}-\frac{h}{c^{*}(l, h, t)} \frac{\partial c^{*}(l, h, t)}{\partial h}=\frac{R(z, t)}{\gamma}>0,
$$

which shows that the propensity to consume out of financial wealth will be always larger than out of "human wealth". This is the generalized PIH as discussed in Wang (2006). The difference decreases from 1 to 0 as liquidity decreases. 


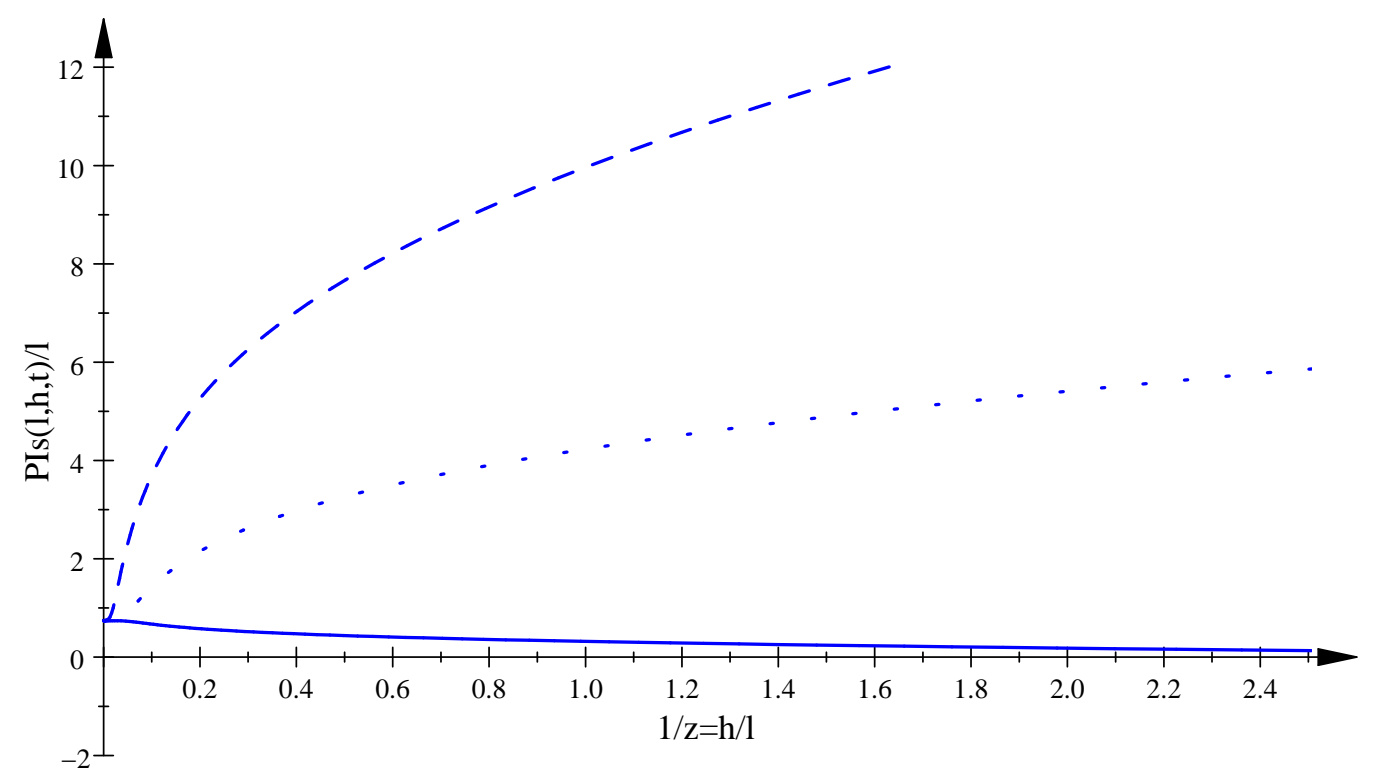

Figure 8: Fractional allocation to the risky liquid asset as a function of $z=l / h$ for different levels of correlation $\rho, \rho=-0.1$ Dashed, $\rho=0.2$ Dotted, $\rho=0.6$ Solid.

\subsection{Allocation choice}

Figure 8 analyzes the allocation to the risky liquid asset per unit of liquid wealth as a function of liquidity, for different values of the correlation between the liquid and the illiquid asset returns. Note that when we increase $h$ the total wealth of the investor increases also. From the figure we see that the allocation to the liquid risky asset increases for negative correlation and also for moderately positive correlation as well, while for high correlation this allocation decreases. For sufficiently low correlation the diversification effect of having the illiquid asset (even if it cannot be traded) increases the optimal allocation to the liquid risky asset, even to the point of borrowing at the risk free asset to invest in the liquid risky asset. Only when the correlation is sufficiently high and the diversification effect of holding the illiquid risky asset diminishes, does the optimal allocation to the liquid risky asset decrease when $h / l$ increases. This analysis indicates that the professor, with his labor income having low correlation with the risky asset (stock market), should invest more in the risky asset than the stock broker, who has a wage highly correlated with the market.

Finally, Figure 9 compares the optimal allocation to the risky asset for different levels of risk aversion. As can be seen from the figure risk aversion has a significant effect on asset allocation: lower risk aversion implying higher 


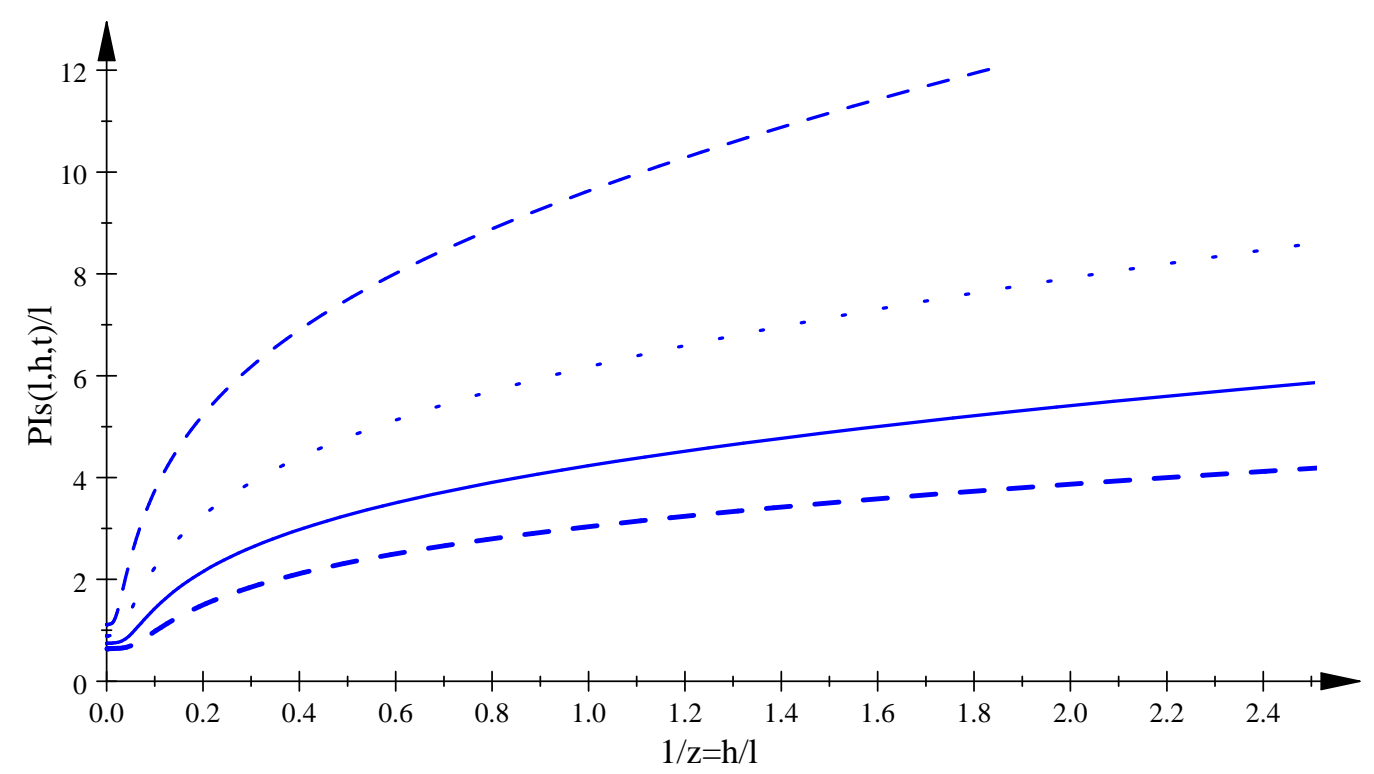

Figure 9: Fractional allocation to the risky liquid asset as a function of $z=l / h$ for different levels of risk aversion $\gamma, \gamma=3.5$ Dashed, $\gamma=3$ Solid, $\gamma=2.5$ Dotted, $\gamma=2$ Bold Dashed.

allocations per unit of liquid wealth to the liquid risky asset. Also, the sensitivity of the allocation to changes in the relative fraction of illiquid wealth to liquid wealth decreases as risk aversion increases.

\subsection{Optimal consumption when the no borrowing con- straint is active at zero wealth}

When the final liquidation of the human capital is sufficiently close and $t>$ $\tau^{*}$, the propensity to consume out of liquid wealth increases inducing the agent to borrow. On the other hand, when the zero wealth boundary is hit the consumption smoothing effect disappears completely because liquidity constraint binds and the optimal choice for the agent is $c=\delta h$, i.e. the agent consumes all the available liquid dividend released from human capital (the wage) without any precautionary saving. Once the zero wealth boundary is hit, it becomes a trap for the agent which is constrained not to participate in the liquid risky asset market and is unable to smooth the effects of income shocks on consumption.

It is remarkable that the introduction of uninsurable income as dividend from illiquid human capital generates optimal consumption policies which are more in line with the empirical findings and a good starting point to interpret 
household consumption choices even in absence of a proper model of credit markets. In particular we observe that this liquidity constrained state is virtually the same found in the analysis of saving and liqudity constraints by Deaton (1991) where it was shown, in a simpler model of consumer choice, that the combination of persistent income and binding liquidity constraints precludes accumulation of assets.

\subsection{The participation puzzle and the zero liquid wealth trap}

As previously discussed, market incompleteness induces an implicit borrowing constraint. This constraint is particularly important for an agent with an high level of human capital and zero liquid wealth. According to our analysis, the optimal policy for an agent expecting that the "duration" of human capital is not too large (smaller than 96.613 years in our choice of parameters) is not to save and not to participate to the financial market, waiting for the liquidation of the proceeds from human capital. This state is a "trap" which can eventually be considered as a potential explanation of the well known participation puzzle. It is quite remarkable that the higher the relative size of human capital with respect to the liquid one, the stronger the effect.

\section{$7 \quad$ Summary and conclusions}

We study the problem of optimal asset allocation in the presence of an illiquid asset. The illiquid asset cannot be traded, but it generates a liquid dividend that can be consumed or invested in liquid assets. This liquid dividend has many interpretations depending on the nature of the illiquid asset. An important application is when the illiquid asset is human wealth and the dividend is labor income. There is a vast literature in economics and finance trying to understand the effect of stochastic labor income on optimal consumption and asset allocation. We obtain closed form solution to this problem in the case of time separable power utility of consumption and terminal wealth.

An important by-product of our analysis is that we derive a valuation procedure for liquid and illiquid assets. In particular, we are able to compute the value that the agent assigns to the illiquid asset, that is, the shadow price of illiquidity. The framework allows, given the preferences of the investor, to value any contingent claim on the illiquid asset or on both the liquid and illiquid asset.

It would be interesting to investigate if the approach we have developed can also be used to solve the optimal asset allocation problem in the presence 
of borrowing and short selling constraints as discussed in general terms by He and Pages (1993) and Cuoco (1997). In particular, it would be interesting to study the effect that these constraints have on the value that the agent assigns to his illiquid asset.

Perhaps the most interesting extension of our analysis from the economic point of view is market equilibrium. If the risky liquid asset is the market portfolio, and the illiquid asset of each agent in the economy is its human wealth, the aggregation problem involves heterogeneous valuations of human wealth holdings of all the agents in the economy. The possibility of asymmetric information effects raises the issue of the impact of moral hazard and adverse selection on such market equilibrium. These extension pose challenging mathematical problems. 


\section{References}

M. Bauer, D. Bernard (2003) SLE martingales and the Virasoro algebra Phys. Lett. B 557, 309-316.

M. Bauer, D. Bernard (2002) SLE growth processes and conformal field theories Phys. Lett. B 543, 135-138.

Bodie, Z., Merton, R.C., and W.F. Samuelson, 1992, Labor supply flexibility and portfolio choice in a life cycle model, Journal of Economic Dynamics and Control 16, 427-449.

Bouchard, B. and Touzi, N. 2009 Weak Dynamic Programming Principle for Viscosity Solutions, preprint.

Campbell, J.Y., A.W. Lo, and A.C. MacKinlay, 1997, The Econometrics of Financial Markets, Princeton University Press, Princeton.

Campbell, J.Y., and L.M. Viceira, 2002. Strategic Asset Allocation: Portfolio Choice for Long Term Investors (Oxford University Press, Oxford).

Calin O.L. , Y. Chen, T. Cosimano, A. A. Himonas, 2005, Solving Asset Pricing Models when the Price-Dividend Function is Analytic, Econometrica, Vol. 73, No. 3, 961-982.

Collin-Dufresne P. and J. Hugonnier, 2006, "On the pricing and hedging of contingent claims in the presence of extraneous risks," Forthcoming Stochastic Processes and Their Applications.

Cox, J. C., and C. F. Huang, 1989, Optimum Consumption and Portfolio Policies When Asset Prices Follow a Diffusion Process, Journal of Economic Theory 49, 33-83.

Cuoco, D., 1997, Optimal Consumption and Equilibrium Prices with Portfolio Constraints and Stochastic Income, Journal of Economic Theory $72,33-73$.

Cvitanic J., Schachermayer W., and H. Wang, 2001, Utility Maximization in Incomplete Markets with Random Endowment, Finance \&5 Stochastics 5, 259-272.

Davis, M. H. A., 1999, Option valuation and hedging with basis risk, in Djaferis, T. E., and Schuck, I. C., eds.: System Theory: Modeling, Analysis and Control (Kluwer: Amsterdam). 
Decamps, M., Goovaerts, M., and W. Schoutens, Asymmetric skew Bessel processes and their applications to finance, Journal of Computational and Applied Mathematics, Volume 186, Issue 1, Jef Teugels, 1 February 2006, Pages 130-147

Detemple, J., and S. Sundaresan, 1999, Nontraded Asset Valuation with Portfolio Constraints: A Binomial Approach, Review of Financial Studies 12, 835-872.

Di Giacinto M., Federico, S. and F. Gozzi, 2010, Pension funds with a minimum guarantee: a stochastic control approach forthcoming $F i$ nance and Stochastics Springer Berlin / Heidelberg.

W. Donoghue. Monotone matrix functions and analytic continuation. Springer, Berlin, Heidelberg, New York, 1974

Dybvig, P., and C. Huang, 1988, Nonnegative Wealth, Absence of Arbitrage, and Feasible Consumption Plans, Review of Financial Studies, vol. 1(4), pp. 377-401.

Dybvig P., and H. Liu, 2004, Lifetime Consumption and Investment: Retirement and Constrained Borrowing, Working paper, AFA 2005 program.

Duffie, D., Fleming, W., Soner, H.M., and T. Zariphopoulou, 1997, Hedging in incomplete markets with HARA utility, Journal of Economic Dynamics and Control 21, 753-782.

El Karoui, N., and M. Jeanblanc, 1998, Optimization of consumption with labor income, Finance \& Stochastics 2, 409-440.

El Karoui, N., and R. Rouge, 2000, Pricing via utility maximization and entropy, Mathematical Finance 10-2, 259-276.

Flavin, M., and T. Yamashita, 2002, Owner-occupied housing and the composition of the household portfolio, The American Economic Review $92,345-363$.

Friedman, M.,1967, A Theory of the Consumption Function, Princeton University Press, Princeton.

Gerber, H.U., and E.S.W. Shiu, 2000, Investing for Retirement: Optimal Capital Growth and Dynamic Asset Allocation, North American Actuarial Journal 4, 42-62. 
Grossman, S., and G. Laroque, 1990, Asset pricing and optimal portfolio choice in the presence of illiquid durable consumption goods, Econometrica 58, 25-52.

He, H., and H.F. Pagès, 1993, Labor income, borrowing constraints, and equilibrium asset prices, Journal of Economic Theory 3, 663-696.

He, H., and N.D. Pearson, 1991, Consumption and portfolio policies with incomplete markets and short-sale constraints: the infinitedimensional case. Journal of Economic Theory 54, 259-304.

Henderson, V., and D. Hobson, 2002, Real Options with Constant Relative Risk Aversion, Journal of Economic Dynamics and Control 27, 329-355.

Henderson, V., 2002, Valuation of claims on nontraded assets using utility maximization, Mathematical Finance 12, 351-373.

Heaton, J. , and D. Lucas, 1997, Market Frictions, Saving Behavior and Portfolio Choice, Macroeconomic Dynamics 1, 76-101.

Heaton, J., and D. Lucas, 2000, Portfolio choice and asset prices the importance of entrepreneurial risk, Journal of Finance 55, 1163-1198.

Henrici, P. Applied and Computational Complex Analysis Wiley

Hodges, S. D. and Neuberger, A., 1980, Optimal replication of contingent claims under transaction costs, Review of Future Markets 8, 222-239.

Hugonnier, J., and D. Kramkov, 2004, Optimal investment with random endowments in incomplete markets, Annals of Applied Probability $14,845-864$.

Jagganathan, R., and N. Kocherlakota, 1996, Why Should Older People Invest Less in Stocks than Younger People?: An Economic Analysis of Financial Planners' Advice, Federal Reserve Bank of Minneapolis Quarterly Review 20, 11-23.

Kahl, M., Liu, J., and F.A. Longstaff, 2003, Paper millionaires how valuable is stock to a stockholder who is restricted from selling it?, Journal of Financial Economics 67, 385-410.

Karatzas, I., and G. Zitkovic, 2003, Optimal Consumption From Investment and Random Endowment in Incomplete Semimartingale Markets, Annals of Probability 31, 1821-1858. 
Koo, H.K., 1995, Consumption and portfolio selection with labor income I: evaluation of human capital, Preprint, Washington University.

Koo, H.K., 1998, Consumption and portfolio selection with labor income a continuous time approach, Mathematical Finance 8, 49-65.

Loewner, C., 1964, On semigroups in analysis and geometry. Bull. Amer. Math. Soc. 701964 1-15.

Markowitz, H. M., 1952, Portfolio Selection, Journal of Finance 7, 7791.

Mayers, D., 1972, Nonmarketable assets and capital market equilibrium under uncertainty, in M.C. Jensen, ed.: Studies in the theory of capital markets (Preager Publishers, New York).

Mayers, D., 1973, Nonmarketable assets and the determination of capital asset prices in the absence of a riskless asset, Journal of Business 46, 258-276.

Merton, R.C., 1969, Lifetime portfolio selection under uncertainty: the continuous-time case, Review of Economics and Statistics 51, 247-257.

Merton, R.C., 1971, Optimum consumption and portfolio rules in a continuous time model, Journal of Economic Theory 3, 373-413.

Merton, R. C., 1992. Continuous-Time Finance (Blackwell).

Musiela, M., and T. Zariphopoulou, 2004, An example of indifference prices under exponential preferences. Finance \& Stochastics 8, 229239.

Samuelson, P.A., 1969, Lifetime Portfolio Selection by Dynamic Stochastic Programming, Review of Economics and Statistics 51, 239246.

Schramm, O., Lawler G., and W. Werner, 2003, Conformal Restriction: The Chordal Case, Journal of the American Mathematical Society V. 16, N. 4, 917-955.

Svensson, L.E.O., and I.M. Werner, 1993, Nontraded assets in incomplete markets : Pricing and portfolio choice, European Economic Review $37,1149-1168$. 
Stanley, H.E., 1999, Scaling, universality, and renormalization: Three pillars of modern critical phenomena. Review of Model Physics 71, S358-S366.

Teplà, L., 2000, Optimal Hedging and Valuation of Nontraded Asset, European Finance Review 4, 231-251.

Viceira, L.M., 2001, Optimal portfolio choice for long-horizon investors with nontradeable labor income, Journal of Finance 56, 433-470.

Wang, N., 2006, Generalizing the Permanent-Income Hypothesis: Revisiting Friedman's Conjecture on Consumption, Journal of Monetary Economics 53, 737-752.

Whittaker, E.T., and G.N. Watson, 1990. A Course in Modern Analysis, (4th ed., Cambridge University Press, England).

Zeldes, S., 1989, Optimal Consumption with Stochastic Income: Deviations from Certainty Equivalence, Quarterly Journal of Economics 104, 275-298.

Zitkovic, 2005, "Utility Maximization with a Stochastic Clock and an Unbounded Random Endowment", Annals of Applied Probability 15 No. 1B, pp 748-777 


\section{Technical Appendix to the paper "Illiquid Assets and Optimal Portfolio Choice" by E. S. Schwartz and C. Tebaldi}

\subsection{The set of admissible strategies}

An admissible strategy is determined by:

- a progressively $\mathcal{F}_{t}$ measurable positive consumption process $\widetilde{c}(t) \geq 0$, $0 \leq t \leq T$ such that

$$
\int_{0}^{t} c(\tau) d \tau<+\infty
$$

- By a set of progressively measurable adapted allocation strategies $\left(\pi_{\tau}^{B}, \pi_{\tau}^{S}\right)$ that verify the integrability conditions

$$
\int_{0}^{T}\left|\pi^{B}\right| r d t+\int_{0}^{T}\left|\pi^{S}\right| \alpha d t+\int_{0}^{T}\left|\pi^{S}\right|^{2} \sigma^{2} d t<\infty .
$$

- The liquid wealth state variable is defined by:

$$
\begin{aligned}
L_{t} & =l \\
L_{\tau} & =\pi_{\tau}^{S}+\pi_{\tau}^{B}, \quad t \leq \tau<T, \\
W_{T} & =\pi_{T}^{S}+\pi_{T}^{B}+H_{T},
\end{aligned}
$$

At the final time $T$ the investor will optimally leave for bequest his total liquid wealth, $W_{T}$ which includes the wealth obtained by liquid investment $L_{T^{-}}=\lim _{t \rightarrow T^{-}} L_{t}$, and the illiquid wealth, $H_{T}$, i.e. $W_{T}=$ $L_{T^{-}}+H_{T}$.

- The set of financeable strategies $\mathcal{A}(l, h, 0)$ given the initial wealth levels $L_{0}=l, H_{0}=h$ is the set of pairs $\left(c(\tau)_{\tau \in[0, T]}, W_{T}^{\pi, c}\right)$ for which there exists an admissible allocation $\left(\pi_{\tau}^{B}, \pi_{\tau}^{S}\right)_{\tau \in[0, T]}$ granting that the dynamic budget equation

$$
d L_{\tau}=\left(r L_{\tau}+\delta H_{\tau}-c_{\tau}\right) d \tau+\pi_{\tau}^{S}\left(\frac{d S_{\tau}}{S_{\tau}}-r d \tau\right) \quad \tau<T
$$

is verified $\forall \tau \in[0, T]$ and the final wealth is nonnegative $W_{T}^{\pi, c} \geq 0$ a.s.

When $\rho \neq \pm 1$ the solvability requirement $W_{T}^{\pi, c} \geq 0$ implies that any admissible strategy must verify $L_{\tau} \geq 0$ a.s. otherwise, in the case of interest, there's a finite probability to end at time $T$ with negative wealth $W_{T}^{\pi, c}<0$. 
This fact implies that the solvability requirement induces a state constraint $L_{\tau} \geq 0$ a.s. $\forall \tau \in[0, T]$, in the stochastic optimization problem. In the next section we analyze the boundary condition implied by this state constraint. Dybvig and Huang (1988) prove that the presence of the nonnegativity constraint is sufficient to grant that the set of admissible strategies is free from arbitrage opportunities.

\subsection{The boundary condition when $h=0$.}

Standard Merton model is recovered if $h=0$. Since the illiquid wealth state variable has a lognormal evolution, the state $H_{t}=0$ is never reached if the initial level $h>0$.

\subsection{The computation of the constrained maximum at the boundary $z=0$}

In order to determine the constrained maximum problem for consumption at $z=0$ :

$$
F_{0}\left(y_{B}(t)\right)=\max _{\delta \geq c \geq 0}\left[-(c-\delta) y_{B}(t)+\frac{c^{1-\gamma}}{1-\gamma}\right]
$$

let us introduce the Lagrangian:

$$
\mathcal{L}\left(p_{1}, p_{2}, c\right)=-(c-\delta) y_{B}(t)+\frac{c^{1-\gamma}}{1-\gamma}-p_{1}(-c)-p_{2}(c-\delta)
$$

the corresponding Kuhn Tucker conditions are:

$$
\begin{aligned}
c^{-\gamma}-y_{B}(t)+p_{1}-p_{2} & =0 \\
p_{1} c & =0 \\
c & \geq 0, p_{1} \leq 0 \\
p_{2}(\delta-c) & =0 \\
c-\delta & \leq 0, p_{2} \geq 0
\end{aligned}
$$

and the possible solutions are:

- $c>0$ and $\delta>c$ then $p_{1}=0$ and $p_{2}=0$;

- $c=0$ and $\delta=c$ then $p_{1}<0$ and $p_{2}>0$;

- $c>0$ and $\delta=c$ then $p_{1}=0$ and $p_{2}>0$; 
The maximization at $t \rightarrow T^{-}$

$$
y_{B}\left(T^{-}\right)=\beta
$$

hence the first order conditions imply:

$$
c^{-\gamma}=\beta-p_{1}+p_{2}
$$

Since $\beta>0, p_{1} \leq 0, p_{2} \geq 0$ then the optimal consumption solution $c>0$ is always strictly positive and thus the constraint $c \geq 0$ is never binding and $p_{1}=0$.

If the constraint $c \leq \delta$ is non-binding, then conditions $c^{-\gamma}=\beta$ and $c<\delta$ are to be verified, hence $\beta>\delta^{-\gamma}$.

On the contrary if

$$
\beta \leq \delta^{-\gamma}
$$

holds, then the slack solution $\left(c^{f}\right)^{-\gamma}=\beta$ implies $c^{f}=\beta^{-\gamma} \geq \delta$ violating the constraint $c \leq \delta$. Hence when $\beta \leq \delta^{-\gamma}$ the constraint $c \leq \delta$ is binding and the solution is:

$$
\begin{aligned}
c & =\delta \\
p_{2}\left(T^{-}\right) & =\delta^{-\gamma}-\beta>0
\end{aligned}
$$

In our analysis it is assumed the condition $\beta \leq \delta^{-\gamma}$ (see Condition A). From an economic point of view this condition is motivated by the assumption that the utility form final liquidation (bequest) is substantially larger than the utility from current consumption.

The maximization for $t<T$

The analytical expression of the boundary $y_{B}(t)$ which is determined by Theorem 2 (see next section) and Kuhn Tucker conditions select the following solutions:

- the solution is internal if

$$
y_{B}(t)=\beta \exp \left[\left(K_{1}+K_{2}-2 K_{3}\right)(T-t)\right]>\delta^{-\gamma}
$$

then $c=y_{B}(t)^{-1 / \gamma}$ and $y_{B}(t)^{-1 / \gamma}<\delta$.

- the constraint binds if

$$
y_{B}(t) \leq \delta^{-\gamma}
$$

and the solution will be:

$$
\begin{aligned}
c & =\delta \\
p_{2}(t) & =\delta^{-\gamma}-y_{B}(t)^{-1 / \gamma}>0
\end{aligned}
$$


Assuming that $\left(K_{1}+K_{2}-2 K_{3}\right) \geq 0, \beta \leq \delta^{-\gamma}$ (see Condition A), the inequality $y_{B}(t) \leq \delta^{-\gamma}$ will hold at any time $t$ such that

$$
\begin{aligned}
(T-t) & \leq \frac{1}{\left(K_{1}+K_{2}-2 K_{3}\right)} \log \left(\frac{\delta^{-\gamma}}{\beta}\right) \\
t & \geqslant \tau^{*} \triangleq \max \left\{T-\frac{1}{\left(K_{1}+K_{2}-2 K_{3}\right)} \log \left(\frac{\delta^{-\gamma}}{\beta}\right), 0\right\}(46)
\end{aligned}
$$

For example in the numerical example is given by:

$$
\begin{aligned}
& \frac{1}{\left(K_{1}+K_{2}-2 K_{3}\right)} \log \left(\frac{\delta^{-\gamma}}{\beta}\right)= \\
& \frac{1}{0.09302222222} \log \left(\frac{0.05^{-3}}{1}\right)=96.613
\end{aligned}
$$

and, since $T=30, \tau^{*}=\max \{0,30-96.613\}$.

The condition $\left(K_{1}+K_{2}-2 K_{3}\right)>0$ imposes that the marginal utility of scaled consumption at zero wealth is decreasing with respect to time. This condition, jointly with $\beta \geq \delta^{-\gamma}$, imply that the liquid value of the illiquid asset grows with time.

In conclusion, on the basis of the above discussion, we can guess that the optimal consumption policy at $z_{t}=0$ is given by:

$$
c_{B}(t)=\left\{\begin{array}{cc}
y_{B}(t)^{-1 / \gamma} \quad t<\tau^{*} \\
\delta \quad \tau^{*} \leq t<T
\end{array}\right.
$$

and imposes to assume a reflecting boundary condition for the state variable $z_{t}$ if the zero wealth boundary is hit before time $\tau^{*}$. In fact it is optimal to consume less than the total dividend thus the agent will save and exit from $z_{t}=0$ state will be instantaneous. On the contrary if the zero boundary is hit after time $\tau^{*}$, it is necessary to assume a killing boundary since it is optimal to consume the whole dividend $c_{t}=\delta$ and wait the final liquidation without saving riskfree asset and without participating to the stock market.

\subsection{Proof of Theorem 2}

The first step toward the statement of the verification result is the determination of the explicit equation for the optimal boundary $y_{B}(t)$.

The ODE for the boundary $y_{B}(t)$ 
From the reduced HJB equation for the primal problem we compute the PDE which describes the evolution of $y(z, t)=V_{z}(z, t)$ getting:

$$
\begin{aligned}
0= & V_{z t}(z, t)+\left(K_{1}+K_{2}-2 K_{3}\right) V_{z}(z, t)+\left(K_{2}+2 K_{4}\right) z V_{z z}(z, t)+ \\
& \left(K_{3} \frac{V_{z}(z, t)^{2}}{z^{2} V_{z z}(z, t)^{2}}+K_{4}\right) z^{2} V_{z z z}(z, t)+F^{\prime}\left(V_{z}(z, t)\right) V_{z z}(z, t),
\end{aligned}
$$

On the other hand admissibility imposes zero investment in the risky asset at the boundary $z=0$, i.e. $\lim _{z \rightarrow 0^{+}} V_{z}(z, t) / V_{z z}(z, t)=0$. Then the limiting expression of the reduced $H J B$ and of PDE (47) imply:

$$
\begin{aligned}
\left\{\frac{\partial y\left(z_{t}, t\right)}{\partial t}+\left(\frac{\partial y(z, t)}{\partial z}\right)_{z=z_{t}}\left(y^{-1 / \gamma}(z, t)-\delta\right)+\left(K_{1}+K_{2}-2 K_{3}\right) y\left(z_{t}, t\right)\right\} d t & =0 \\
\left\{d z_{t}+\left(\delta-y^{-1 / \gamma}(z, t)\right)\right\} d t & =0 \\
\left\{\frac{\partial y\left(z_{t}, t\right)}{\partial t} d t+\left(\frac{\partial y(z, t)}{\partial z}\right)_{z=z_{t}} d z_{t}+\left(K_{1}+K_{2}-2 K_{3}\right) y\left(z_{t}, t\right) d t\right\} & =0 \\
\left\{d y\left(z_{t}, t\right)+\left(K_{1}+K_{2}-2 K_{3}\right) y\left(z_{t}, t\right) d t\right\} & =0
\end{aligned}
$$

Hence the unique deterministic evolution of the boundary point $y_{B}(t)=$ $\lim _{z \rightarrow 0^{+}} V_{z}(z, t)$ which can be obtained as a limiting expression of the reduced HJB in the region $\mathcal{D}_{t}$ under the regularity Assumption $A$ is defined by the ODE:

$$
\frac{d}{d t} y_{B}(t)=-\left(K_{1}+K_{2}-2 K_{3}\right) y_{B}(t)
$$

Assumption of continuity w.r.t to time (see Condition A) of $V_{z}(z, t)$ as $t \rightarrow$ $T^{-}$determines the boundary condition:

$$
y_{B}(T)=\beta
$$

Observe that in the case $y^{-1 / \gamma}(z, t)<\delta$ the constraint on maximum scaled consumption determined by the No Borrowing constraint is not active and $c_{B}(t)=y_{B}^{-1 / \gamma}(0, t)$, thus the optimal consumption feedback policy is right continuous in the limit $z \rightarrow 0^{+}$. Consider now the case $y_{B}^{-1 / \gamma}(0, t)>\delta$, then at $z=0$, the constraint on maximum consumption is active, and the optimal scaled consumption feedback function is discontinuous, in fact:

$$
c_{B}(t)=y_{B}^{-1 / \gamma}(t)=\lim _{z \rightarrow 0^{+}} V_{z}^{-1 / \gamma}(z, t)>\delta
$$

which implies that in correspondence to the hitting of the $z=0$ barrier the optimal level of consumption must drop discontinuously to $\delta$ and the diffusion process has to be killed collapsing onto the state $z_{t}=0, c=\delta$. 
The verification that $V(z, t)$ solves the reduced maximization problem.

To prove that the function $V(z, t)$ defined by eq.s $(28,29)$ is the solution to the reduced primal maximization problem we apply the dual verification result stated in Theorem 5.1 in Pham and Mnif (2001) which extended to the incomplete market setup the procedure of He and Pagès (1991) and El Karoui and Jeanblanc (1997).

The first step of this procedure is the formulation and the solution of the dual stochastic mixed regular/singular minimization problem. In the present framework it turns possible to solve the dual minimization problem using a standard dynamic programming verification argument which shows that the solution to the HJB variational inequality (16):

$$
\begin{aligned}
& \widetilde{V}(y, t) \quad y \in \mathcal{D}_{t} \\
& \widetilde{V}\left(y_{B}(t), t\right) \triangleq \lim _{y \rightarrow y_{B}(t)^{-}} \widetilde{V}(y, t) \quad t \in\left[0, \tau^{*}\right) \\
& \widetilde{V}\left(\delta^{-\gamma}, t\right) \triangleq V^{B}(t) \quad t \in\left[\tau^{*}, T\right)
\end{aligned}
$$

verifies the dynamic programming principle:

$$
\begin{aligned}
\widetilde{V}(y, t) & =\inf _{Y \in \mathcal{Y}^{l o c}} E_{(t, y)}^{\mathbb{P}}\left[e^{-K_{1}(T-t)}\left(-\beta^{1 / \gamma} \frac{\left(Y_{T}\right)^{b}}{b}+Y_{T}\right)+\int_{t}^{T} e_{s}^{-K_{1}(s-t)} Y_{s} F\left(Y_{s}\right) d s\right] \\
\widetilde{V}(y, T) & =y-\beta^{1 / \gamma} \frac{y^{b}}{b} \quad y \in \mathcal{D}_{T} \triangleq(0, \beta]
\end{aligned}
$$

The general characterization of the dual domain $\mathcal{Y}^{l o c}$ granting existence of the minimizer has been given in Karatzas and Zitkovic (2003) and proves that the admissible state processes $Y_{t}$ are in the class of nonnegative supermartingale processes. In the present Markovian framework it is not restrictive to assume that the set of admissible dual controls is parametrized as follows:

$$
\begin{aligned}
\frac{d Y_{t}}{Y_{t}} & =\frac{d M_{t}}{M_{t}}+\mu\left(Y_{t}, t\right) d t+\sigma_{1}^{S D F}\left(Y_{t}, t\right) d W_{t}^{1}+\sigma_{2}^{S D F}\left(Y_{t}, t\right) d W_{t}^{2} \\
y_{0} & =y \in\left(0, y_{B}(0)\right) \quad t \in[0, \zeta \wedge T)
\end{aligned}
$$

where the coefficients $\mu(y, t)$ and $\sigma_{1}^{S D F}(y, t), \sigma_{2}^{S D F}(y, t)$ are assumed to be bounded. The process $Y_{t}$ is factorized as $Y_{t}=M_{t} Y_{t}^{f}$, where $Y_{t}^{f}$ is a regular process and $M_{t}$ is a singular process which is used to implement the reflecting boundary conditions, while $\zeta(\omega)$ is an absorption stopping time for the diffusion at $y_{B}(t)$ and $\zeta \wedge T=\min \{\zeta, T\}$. The stopping time $\zeta(\omega)>0$ has to be considered as part of the control specification and is necessary to take into account the possible admissible strategies corresponding to a diffusion which exits from the state space $\left(0, y_{B}(t)\right)$ at $\zeta(\omega)$ and is absorbed 
to the state $y=\delta^{-\gamma}>y_{B}(t)$ where it remains for the entire period $[\zeta, T)$ : $\lim _{t \rightarrow \zeta^{-}} y_{t}=y_{B}(\zeta)$, and $y_{t}(\omega)=\delta^{-\gamma}, \zeta(\omega) \leq t<T$.

The construction of the feedback expression of the optimal dual state process, which is denoted with the small letter $y_{t}$ is detailed in Proposition 3 .

Under the smoothness assumptions implied by Condition A, application of the generalized Ito Lemma implies:

$$
\begin{aligned}
& \widetilde{V}\left(y_{T}, T\right)+\int_{t}^{T} e^{-K_{1}(s-t)} y_{s} F\left(y_{s}\right) d s \\
= & \widetilde{V}\left(y_{t}, t\right)+\int_{t}^{T} \operatorname{HJB}[\widetilde{V}(y, t)] d s-\int_{t}^{\tau^{*}} y_{t} \widetilde{V}_{y}\left(y_{t}, t\right) \frac{d L^{y_{t}}\left(y_{B}(t)\right)}{y_{B}(t)} \\
& +\int_{t}^{T} e^{-K_{1}(s-t)} y_{s} F\left(y_{s}\right) d s+\int_{t}^{T \wedge \tau_{B}(\omega)} y_{t} \widetilde{V}_{y}\left(y_{t}, t\right)\left(\sqrt{2 K_{3}} d W_{t}^{1}+\sqrt{2 K_{4}} \widetilde{R}\left(y_{t}, t\right) d W_{t}^{2}\right) \\
= & \widetilde{V}\left(y_{t}, t\right)+\int_{t}^{T} y_{t} \widetilde{V}_{y}\left(y_{t}, t\right)\left(\sqrt{2 K_{3}} d W_{t}^{1}+\sqrt{2 K_{4}} \widetilde{R}\left(y_{t}, t\right) d W_{t}^{2}\right)
\end{aligned}
$$

and taking the conditional expectation it is proved that $\widetilde{V}\left(y_{t}, t\right)$ solution to the HJB variational inequality (16) verifies eq.(49).

Having determined the solution to the dual problem, all the hypotheses are verified and the dual verification result of Theorem 5.1 in Pham and Mnif (2001) can be applied. This implies that the duality relations $(12,28)$ hold, hence $V(z, t)$ is uniquely determined by the dual value function $\widetilde{V}(y, t)(48)$. Given uniqueness of the solution $V(z, t)$ to the reduced problem, then the value function $V(l, h, t)=h^{1-\gamma} V(z, t)$ solves the original stochastic optimization problem.

\subsection{Proof of Proposition 3}

Complementary slackness condition at the saddle point imply that the unique solution for $\mu(y, t)$ and $\sigma_{1}^{S D F}(y, t)$ is given by:

$$
\begin{aligned}
\mu(y, t) & =-\left(K_{1}+K_{2}-2 K_{3}\right) \\
\sigma_{1}^{S D F}(y, t) & =\sqrt{2 K_{3}}
\end{aligned}
$$

The optimal expression of the regular process $Y_{t}^{f}$ requires the determination of the volatility coefficient $\sigma_{2}^{S D F}(y, t)$. Following the argument of $\mathrm{He}$ and Pearson (1991) and the computation detailed in the proof of Corollary 6, it is verified that the term

$$
\sigma_{2}^{S D F}(y, t)=\sqrt{2 K_{4}} \widetilde{R}(y, t)=\sqrt{2 K_{4}} \frac{\widetilde{V}_{y}(y, t)}{y \widetilde{V}_{y y}(y, t)}
$$


can be interpreted as the feedback expression obtained by the minimization of the uninsurable component of risk that drives the shadow prices of consumption. The optimal expressions for the singular controls $M_{t}$ and $\zeta$ are uniquely selected by the condition that the system of optimal dual shadow prices is compatible with the borrowing constraint in the primal problem. The dynamics and the optimal policies when the constraint is active can be derived by constraining the state space of the diffusion to the region $\left(0, y_{B}(t)\right)$. Then the behavior of the diffusion must be completed with a classification of the boundary behavior when the process approaches the boundaries $y_{t} \rightarrow 0$ and $y_{t} \rightarrow y_{B}(t)$. This can be done through an application of the Feller classification of one dimensional diffusion boundaries.

Condition (20) and the limiting expression of the coefficients $(50,51)$ imply:

Proposition 8 Assume Condition $\boldsymbol{A}$ and let condition (20) hold, then starting from $y \in\left(0, y_{B}(t)\right) t \leq T$, the probability that $y_{t}$ reaches $y=0$ is zero, while there's a finite probability to reach $y_{B}(t)$. Hence according to the Feller classification $y=0$ is a "natural boundary" while $y_{B}(t)$ is "regular".

Proof Straightforward application of Feller classification criteria produces the result. End Proof

According to Feller theory, the specification of boundary conditions is required only for regular boundaries, hence the definition of the process $y_{t}$ must be completed with the determination of the boundary conditions at $y_{B}(t)$.

The type of boundary condition to be imposed as $y_{t} \rightarrow y_{B}(t)$ are determined by the lateral conditions on the dual value function which were assumed in the verification theorem or, equivalently, by the analysis of the admissible optimal allocation and consumption policies for an agent in the limiting situation of $z \rightarrow 0$. The classification goes as follows:

- Consider first the Regime $I, t<\tau^{*}$. The optimal control policy is consistent with an instantaneously reflecting boundary condition for $y_{t}$.

If $K_{3} \neq 0$, then $\lim _{y \rightarrow y_{B}^{-}(t)} \sigma^{2}(y, t)=2 K_{3} y_{B}^{2}(t) \neq 0$ and a singular control action must be exerted to "regulate" the process $y_{t}$ and constrain its evolution to stay within the admissible state space. Repeating the considerations of He and Pagès (1991) and extending their approach to the incomplete market setup, the candidate optimal dynamics for the 
shadow prices process when $t<\tau^{*}$ is given by:

$$
\begin{aligned}
\frac{d y_{t}}{y_{t}} & =\frac{d M_{t}^{*}}{M_{t}^{*}}-\left(K_{1}+K_{2}-2 K_{3}\right) d t+\sqrt{2 K_{3}} d W_{t}^{1}+\sqrt{2 K_{4}} d W_{t}^{2} \\
y_{t} & \in\left(0, y_{B}(t)\right] \quad t<\tau^{*} \\
\int z_{t} Y_{t}^{f} d M_{t}^{*} & =0 \text { if } \quad y_{t} \in \mathcal{D}_{t}=\left(0, y_{B}(t)\right)
\end{aligned}
$$

The last equation specifies that the process $M_{t}^{*}$ remains constant if $y_{t} \in \mathcal{D}_{t}$, while it has a finite instantaneous variation when the critical boundary $y_{B}(t)$ is hit. In fact the computation of the (generalized) Ito differential for the process $y_{t}=\min \left\{y_{B}(t), y_{t}^{f}\right\}$ where $y_{t}^{f}$ is the unconstrained process produces:

$$
\frac{d y_{t}}{y_{t}}=\mu\left(y_{t}, t\right) \mathbb{I}_{\left\{y_{t}<y_{B}(t)\right\}} d t+\sigma\left(y_{t}, t\right) \mathbb{I}_{\left\{y_{t}<y_{B}(t)\right\}} d \widetilde{W}_{t}-\frac{1}{2} \frac{d L^{y_{B}(t)}\left(y_{t}\right)}{y_{B}(t)}
$$

where $\mathbb{I}_{\left\{y_{t}<y_{B}(t)\right\}}$ denotes the indicator function of the event $\left\{y_{t}<y_{B}(t)\right\}$. The term $L^{y_{t}}\left(y_{B}(t)\right)$ is the local time of the process $y_{t}$ at the boundary $y_{B}(t)$. It is required to take into account that the function $g(x)=\max \left\{y_{B}(t), x\right\}$ is not differentiable at $y_{B}(t)$. Hence drift and diffusion coefficients describe the local evolution of the process in $y_{t} \in \mathcal{D}_{t}$, while the additional contribution:

$$
\frac{d M_{t}^{*}}{M_{t}^{*}}=-\frac{1}{2} \frac{d L^{y_{B}(t)}\left(y_{t}\right)}{y_{B}(t)}
$$

is the singular component granting that the process $y_{t}$ is reflected at the upper critical boundary $y_{B}(t)$. The possibility to find a pair of processes $\left(M_{t}, y_{t}\right)$ which implement the reflecting boundary condition or, in a more mathematical terminology, which solve the Skorohod problem, is granted by an application of the Th. 5.4 pg. 83 of Oksendal and Sulem (2006).

As explained in He and Pagès (1991) the process $M_{t}^{*}$ determines the contribution to the shadow prices generated by the liquidity constraint.

If $K_{3}=0$ then by condition $(20) \sigma^{2}(y, t) \rightarrow 0$ as $y_{t} \rightarrow y_{B}(t)$ then the optimal shadow price process is given by:

$$
\frac{d y_{t}}{y_{t}}=-\left(K_{1}+K_{2}\right) d t+\sqrt{2 K_{4}} \frac{\widetilde{V}_{y}\left(y_{t}, t\right)}{y_{t} \widetilde{V}_{y y}\left(y_{t}, t\right)} d W_{t}^{2}
$$

When the process $y_{t}$ reaches the barrier $y_{B}(t)$, it is instantaneously reflected back into the region $y<y_{B}(t)$ since the conditions on parameters grant that $\left(K_{1}+K_{2}\right)>0$. No singular control policy is required. 
- Regime $I I, t \geq \tau^{*}$. Let $\tau_{t}(\omega)$ be the first time the process started at time $t$ hits the barrier $y_{B}\left(t^{\prime}\right)$ after time $\tau^{*}, t^{\prime}>\tau^{*}$ :

$$
\tau^{B}(\omega)=\inf \left\{\tau \geq 0: \quad \tau^{*}<\tau \leq T, \quad \lim _{\tau^{\prime} \rightarrow \tau^{-}} y_{\tau^{\prime}}(\omega)=y_{B}(\tau)\right\}
$$

with the convention that inf $\{\varnothing\}=T$. Then the diffusion $y_{t}$ is still determined by eq.(52) but it is stopped at $\tau^{B}(\omega)$. Hence the state space of the diffusion is given by

$$
y_{t}(\omega) \in\left(0, y_{B}(t)\right) \quad \tau^{*} \leq t<\tau^{B}(\omega)
$$

the process $y_{t}$ is killed when the boundary is hit and $y_{t}$ jumps to the "cemetery" state $y=\delta^{-\gamma}$ where it remains until the final liquidation time $T$ :

$$
\begin{aligned}
y_{t}(\omega) & =\delta^{-\gamma} \quad \tau^{B}(\omega) \leq t<T \\
y_{T}(\omega) & =\beta
\end{aligned}
$$

Hence in Regime II the state space for the process of $y_{t}, \tau^{*}<t<T$ is given by the set $\mathcal{D}_{t} \cup\left\{\delta^{-\gamma}\right\}=\left(0, y_{B}(t)\right) \cup\left\{\delta^{-\gamma}\right\}$ and $\left\{\delta^{-\gamma}\right\}$ is an isolated point since $\delta^{-\gamma}>y_{B}(t)$.

\subsection{Proof of Theorem 5}

The next Lemma proves the first part of the Theorem.

Lemma 9 Consider the following rescaling of variables:

$$
\begin{aligned}
\widetilde{\delta}_{1}(T-t) & =\delta \exp \left[\frac{\left(K_{1}+K_{2}-2 K_{3}\right)+\gamma\left(K_{2}+2 K_{4}\right)}{\gamma}(T-t)\right] \\
\widetilde{c}_{t} & =c_{t} \exp \left[\frac{\left(K_{1}+K_{2}-2 K_{3}\right)}{\gamma}(T-t)\right] \\
\widetilde{z}(\widetilde{c}, T-t) & =z(c, t) \exp \left[\left(K_{2}+2 K_{4}\right)(T-t)\right]
\end{aligned}
$$

Then scaled consumption evolves like a time changed Bessel process with state space $[1,+\infty)$ and boundary 1 :

$$
\begin{aligned}
d \widetilde{c}_{\varphi_{t}} & =\frac{1}{2}(1+\gamma) \frac{d \varphi_{t}}{\widetilde{c}_{\varphi_{t}}}+d \widetilde{B}_{\varphi_{t}}+\frac{1}{2} d L_{\varphi_{t}}^{1}\left(\widetilde{c}_{\varphi_{t}}\right) \\
\varphi_{t} & =2 \int_{0}^{t} \nu\left(\widetilde{c}_{s}, T-s\right) d s \quad t \leq \tau^{B}(\omega) \\
\nu(\widetilde{c}, T-s) & =\frac{\widetilde{c}^{2}}{\gamma^{2}}\left[K_{3}+K_{4}\left(\frac{\widetilde{z}(\widetilde{c}, T-s)}{\widetilde{c} \widetilde{z}_{\widetilde{c}}(\widetilde{c}, T-s)}\right)^{2}\right]
\end{aligned}
$$


The time change does not change the classification of boundaries, hence the boundary 1 is reflecting for $0<t<\tau^{B}(\omega)$ and killing at the first hitting time $\tau^{B}(\omega)>\tau^{*}$ when $\widetilde{c}_{\tau^{B}(\omega)}$ jumps to the "cemetery state" $\delta \exp \left[-\frac{\left(K_{1}+K_{2}-2 K_{3}\right)}{\gamma}(T-t)\right], \tau^{B}(\omega) \leq t<T$.

Proof Recall that a stochastic time change is the transformation induced on a diffusion process by changing the "clock", i.e. introducing a new (stochastic) parametrization of time. We refer to standard probability textbooks for the rigorous definition of the time change transformation, see e.g. Rogers and Williams (2000), and simply recall the basic property which is relevant to state our result: consider a diffusion process described by the stochastic differential equation

$$
d X_{t}=\mu\left(X_{t}\right) d t+\sigma\left(X_{t}\right) d B_{t}
$$

then a stochastic time change is an increasing positive right continuous process $t \rightarrow \Phi_{t}$. Assuming $\Phi_{t}$ to have an absolutely continuous density, say $n\left(X_{t}\right)$, such that:

$$
t \rightarrow \Phi_{t}=\int_{0}^{t} n\left(X_{s}\right) d s
$$

then a time change of the diffusion process $X_{t}$ is a new diffusion process $Y_{\Phi}$, such that at any time $t, \Phi_{t}$ is a stopping time for $Y_{\Phi}$ and

$$
Y_{\Phi_{t}}=X_{t}
$$

almost surely. Then, using the scaling property of the Brownian motion, it is possible to prove that the stochastic differential equation for the time changed diffusion process $Y_{\Phi}$ is given by:

$$
d Y_{\Phi}=\frac{\mu\left(Y_{\Phi}\right)}{n\left(Y_{\Phi}\right)} d \Phi+\frac{\sigma\left(Y_{\Phi}\right)}{\sqrt{n\left(Y_{\Phi}\right)}} d \widetilde{B}_{\Phi}
$$

where $\widetilde{B}_{\Phi}$ is a Brownian Motion with respect to the time parameter $\Phi$. Direct application of the above formula assuming $\Phi_{t}=\varphi_{t}$ and $n\left(\widetilde{c}_{t}\right)=\nu\left(\widetilde{c}_{t}, T-t\right)$, proves that the optimal consumption process can be time changed to a Bessel process. The time changed process with state space limited to $[1,+\infty)$ can be considered a skewed Bessel process. The formal construction and definition of this process can be found in Decamps, Goovaerts and Schoutens (2004, 2006). End Proof

In order to prove the second part of the theorem, let us briefly recall some basic properties of conformal mappings: 
Lemma 10 (Donoghue 1974) Let $\mathbb{H}=\{Z=\operatorname{Re} Z+i \operatorname{Im} Z: \operatorname{Im} Z>0\} a$ conformal mappings $\phi: \mathbb{H} \rightarrow \overline{\mathbb{H}}$, i.e. an analytic univalent mapping of the upper half complex plane in itself has the following properties

1. the Pick integral representation:

$$
\phi(Z)=b_{0}+b_{1} Z+\int_{\mathbb{R}} \frac{1+t Z}{t-Z} \nu(d t)
$$

$b_{0}=\bar{b}_{0}, b_{1} \geq 0$ and $\nu$ is a finite nonnegative Borel measure on $\mathbb{R} ;$

2. its Laurent expansion at infinity is given by:

$$
\phi(Z) \stackrel{Z \rightarrow+\infty}{\simeq} b_{0}+b_{1} Z+\sum_{n=1}^{+\infty} b_{n} Z^{-n}
$$

with $b_{n} \in \mathbb{R}$. A simple transformation allows to introduce the so called hydrodynamic normalization for the map $\phi$. Each $\phi$ can be reduced to the form:

$$
\phi(Z) \stackrel{Z \rightarrow^{+\infty}}{\simeq} Z+\sum_{n=1}^{+\infty} b_{n}^{\prime} Z^{-n}
$$

$b_{n}^{\prime} \in \mathbb{R}$.

3. Conformal functions forms a semigroup with respect to composition, i.e. if $\phi_{1}, \phi_{2}$ are conformal maps, then $\phi_{1} \circ \phi_{2}$ is conformal.

Let $\Phi_{\mathbb{H}}$ be the set of inverse conformal transformations. Also this set of transformations forms a semigroup: to each pair of elements $g_{1}, g_{2} \in \Phi_{\mathbb{H}}$ swe can associate the map $g_{3}=\left(g_{1}^{-1} \circ g_{2}^{-1}\right)^{-1}$. Lagrange inversion formula proves that also the element of $g \in \Phi_{\mathbb{H}}$ has a Laurent expansion (54) which can be hydrodynamically normalized.

Proof of the regularity result: $\widetilde{z}(\widetilde{c}, \tau) \in \Phi_{\mathbb{H}}, \forall \tau \in[0, T]$

The proof of the second part of the theorem is easily obtained as an extension of an argument discussed in Bernard and Bauer (2002, 2003). These authors prove that the Bessel process induces a stochastic flow in the space of maps belonging to $\Phi_{\mathbb{H}}$ and hydrodynamically normalized at $\infty$. The argument is essentially the following: let $F(c, T)$ be a germ of an analytic function with (real) analytic expansion as $c \rightarrow \infty$

$$
F(c)=\sum_{n=0}^{+\infty} f_{n} c^{1-n}
$$


then for any $g \in \Phi_{\mathbb{H}}$, it is possible to define an operator $\gamma_{g}$ acting on $F$

$$
\gamma_{g} F=F \circ g
$$

This set of operators forms a semigroup with a composition law $\gamma_{g_{1}} \cdot \gamma_{g_{2}}=\gamma_{g_{3}}$ where $g_{3}=\left(g_{1}^{-1} \circ g_{2}^{-1}\right)^{-1}$.

Consider the SDE for the Bessel process:

$$
d c_{t}=\left(\frac{1}{2}+d\right) \frac{d t}{c_{t}}+d B_{t} \quad c_{0}=c,
$$

the random stochastic flow $c_{t}(c, \omega)$ associated to the SDE is the application which maps the initial condition $c$ to the terminal one $c_{t}$ along path $\omega$. The random stochastic flow defined by the Bessel SDE can be expanded at $c \rightarrow+\infty$ :

$$
c_{t}(c, \omega) \stackrel{c \rightarrow+\infty}{\simeq} c+\gamma_{0}(t)+\sum_{n=2}^{+\infty} \gamma_{n}(t) c^{1-n}
$$

where $\gamma_{0}(t)=B_{t}(\omega)$ and $\gamma_{n}(t)$ are random processes independent from $c_{0}$. Their stochastic evolution is uniquely determined inserting (56) in the SDE (55) imposing that the SDE holds independently from the value of $c$. Then the following set of equations is obtained:

$$
\sum_{n=0}^{+\infty} d \gamma_{n}(t) c_{0}^{1-n}=\sum_{n=0}^{+\infty} p_{n}\left(\left\{\gamma_{n}(t)\right\}\right) c_{0}^{1-n} d t+d B_{t}
$$

where $p_{n}\left(\left\{\gamma_{n}(t)\right\}\right)$ is a polynomial in $\gamma_{n}(t)$ defined by

$$
\begin{aligned}
c_{t}^{-1}\left(c_{0}\right) & =\sum_{n=0}^{+\infty} p_{n}\left(\left\{\gamma_{n}(t)\right\}\right) c_{0}^{1-n}, \quad p_{1}=0, p_{2}=1, \\
p_{n}\left(\left\{\gamma_{n}(t)\right\}\right) & =-\sum_{i=1}^{n-2} \gamma_{i}(t) p_{n-i}\left(\left\{\gamma_{n}(t)\right\}\right)
\end{aligned}
$$

The possibility to expand the stochastic flow of a Bessel process in such a series has an algebraic motivation: in fact the Bessel generator

$$
\mathcal{A}=\left(\frac{1}{c} \frac{\partial}{\partial c}+\left(\frac{1}{2}+d\right) \frac{\partial^{2}}{\partial c^{2}}\right)
$$

can be written like an element of the Virasoro algebra. In fact $\mathcal{A}$ can be written as:

$$
\mathcal{A}=\left(-\left(\frac{1}{2}+d\right) L_{2}+\frac{1}{2} L_{1}^{2}\right)
$$


where $L_{n}$ denote the generators of this algebra which are the family of differential operators

$$
L_{n}=-c^{1-n} \frac{\partial}{\partial c} \quad n \geq 0
$$

and are well known to be the infinitesimal generators of the conformal transformations. Each generator $L_{n}$ spans the subspace of conformal maps corresponding to the coefficient $\gamma_{n}(t) c^{1-n}$. The Virasoro Algebra generators are known to correspond to the infinitesimal generators for a semigroup of composition operators within the class of inverse conformal functions. Similarly, application of Ito lemma implies:

$$
d F\left(c_{t}\left(c_{0}\right)\right)=\mathcal{A} F\left(c_{t}\left(c_{0}\right)\right) d t+F^{\prime}\left(c_{t}\left(c_{0}\right)\right) d B_{t}
$$

which can be rewritten in symbolic notation as:

$$
d \gamma_{c_{t}} \cdot F=\gamma_{c_{t}}\left\{\left[-\left(\frac{1}{2}+d\right) L_{2}+\frac{1}{2} L_{1}^{2}\right] d t-L_{2} d B_{t}\right\} \cdot F
$$

and this formula shows that the stochastic flow $\gamma_{c_{t}}$ generated by $\mathcal{A}$ is a one parameter stochastic sub-semigroup acting on $F$ by composition as follows:

$$
\gamma_{c_{\tau}} \cdot F=F\left(c_{\tau}\left(c_{0}, \omega\right)\right)
$$

and the stochastic flow of a Bessel process can be interpreted as a one parameter sub-semigroup of the semigroup generated by the Virasoro Algebra of inverse conformal mappings $\gamma_{g}, g \in \Phi_{\mathbb{H}}$ with an analytic expansion of the form (54) and hydrodynamic normalization at $\infty$.

The stochastic evolution of $F\left(c_{\tau}\left(c_{0}, \omega\right), \tau\right)$ can be rewritten as a stochastic evolution for the coefficients $f_{n}$ of its analytic expansion of $F$ at $\infty$. Correspondingly the martingale condition:

$$
\frac{\partial}{\partial \tau} F(c, \tau)+\mathcal{A} F(c, \tau)=0
$$

determines a deterministic evolution equation in the manifold of inverse conformal functions. These functions are parametrized by the asymptotic coefficients $f_{n}(\tau)$ such that $F(c, \tau) \stackrel{c \rightarrow+\infty}{\simeq} \sum_{n} f_{n}(\tau) c^{1-n}$. In addition, a group theoretical construction analyzed by Bauer and Bernard (2003) shows that the equations which determine $f_{n}(\tau)$ have a hierarchical triangular structure. Hence, provided that $F(c, 0) \in \Phi_{\mathbb{H}}$, the solution to the boundary value problem generated by the martingale problem (57) can be reduced to an infinite hierarchy of ODE for the coefficients $f_{n}(\tau)$ which parametrize a family of inverse conformal mappings. 
It will be proved that the same property holds for the non linear boundary value problem (34).

First of all it is easy to show that (34) can be restated as a boundary value problem in a space of inverse conformal maps hydrodynamically normalized at $\widetilde{c}=+\infty$. In fact observe that without loss of generality, the change of variables: $\widetilde{c} \rightarrow \widetilde{c}=b_{0}(\tau)+b_{1}(\tau) \widetilde{c}$ maps the original problem to the following simplified version:

$$
\begin{gathered}
-\frac{\partial}{\partial \tau} \widetilde{z}(\widetilde{c}, \tau)+\left(\frac{(1+\gamma)}{2} \frac{1}{\widetilde{c}} \widetilde{z}_{c}(\widetilde{c}, \tau)+\frac{1}{2} \widetilde{z}_{c c}(\widetilde{c}, \tau)\right) \nu(\widetilde{c}, \tau)=0 \\
\nu(\widetilde{c}, \tau)=2 \frac{\widetilde{c}^{2}}{\gamma^{2}}\left[K_{3}+K_{4}\left(\frac{\widetilde{z}(\widetilde{c}, \tau)}{\widetilde{c} \widetilde{c}(\widetilde{c}, \tau)}\right)^{2}\right] \\
\widetilde{z}(\widetilde{c}, 0)=\widetilde{c} \\
\lim _{\widetilde{c} \rightarrow \widetilde{c}_{B}^{+}=\widetilde{c}^{\prime}(1)} \widetilde{z}(\widetilde{c}, \tau)=0 \quad \lim _{\widetilde{c} \rightarrow \widetilde{c}_{B}^{+}=\widetilde{c}^{\prime}(1)} \widetilde{z_{c}}(\widetilde{c}, \tau)=0 \quad \lim _{\widetilde{c} \rightarrow \widetilde{c}_{B}^{+}=\widetilde{c}^{\prime}(1)} \frac{\widetilde{z}(\widetilde{c}, \tau)}{\widetilde{c} \widetilde{z}_{c}(\widetilde{c}, \tau)}=0
\end{gathered}
$$

Solutions to (58) can be assumed to be hydrodynamically normalized mappings in $\Phi_{\mathbb{H}}$. Direct substitution of the series expansion solution (25) for $\widetilde{z}(\widetilde{c}, \tau)$ in eq.s $(58)$ shows that the solution family has an asymptotic expansion compatible with the property that the functions $\widetilde{z}(\widetilde{c}, \tau)$ are inverse conformal functions, i.e. $\widetilde{z}(\widetilde{c}, \tau) \in \Phi_{\mathbb{H}}$ and suggests to interpret the boundary value problem eq.(58) as a martingale problem with respect to time changed Bessel process. In fact eq.s (58) and (57) are related by a simple change of time scale. The martingale problem defined by eq.s (58) is given by:

$$
\widetilde{C}_{t}^{f}=f\left(\widetilde{c}_{t}\right)-f\left(\widetilde{c}_{0}\right)-\int_{0}^{t} \mathcal{A} f\left(\widetilde{c}_{s}\right) \nu\left(\widetilde{c}_{s}, T-s\right) d s \quad \mathcal{F}_{t} \text {-martingale }
$$

and defining the increasing process:

$$
a_{t}=\int_{0}^{t} \nu^{-1}\left(\widetilde{c}_{s}, T-s\right) d s,
$$

then

$$
\alpha_{t}=\inf \left\{u: a_{u}>t\right\}
$$

is a stopping time. The change of variables $\widetilde{c}_{t}=\kappa_{\alpha_{t}}$ implies

$$
C_{\alpha_{t}}^{f}=f\left(\kappa_{\alpha_{t}}\right)-f\left(\kappa_{0}\right)-\int_{0}^{\alpha_{t}} \mathcal{A} f\left(\kappa_{s}\right) d s \quad \mathcal{F}_{\alpha_{t}} \text { - martingale }
$$

which is the martingale problem for a standard Bessel process.

Since the initial element of the solution family is an element of $\Phi_{\mathbb{H}}$, $\widetilde{z}(\widetilde{c}, 0)=\widetilde{c}$, provided the existence of a bounded stochastic time density 
$\nu(\widetilde{c}, \tau)$ which maps (59) onto (60), it is possible to conclude that the functions which solve the martingale problem generated by eq.(58) are still inverse conformal functions, i.e. belong to $\Phi_{\mathbb{H}}$.

The characterization of the time change is complicated by the fact that the stochastic time density $\nu(\widetilde{c}, \tau)$ is determined by the solution $\widetilde{z}(\widetilde{c}, \tau)$, i.e. it is in feedback form. Thanks to the assumption $K_{3} \neq 0$, the function $\nu(\widetilde{c}, \tau)$ is bounded and different from 0 , if and only if $E_{\widetilde{z}} \triangleq(\widetilde{z}(\widetilde{c}, \tau) / \widetilde{c} \widetilde{c}(\widetilde{c}, \tau))$ is bounded. Now we prove that $E_{\widetilde{z}}$ is bounded for any $\widetilde{z} \in \Phi_{\mathbb{H}}$ which solves (58). By definition $E_{\widetilde{z}}$ is positive, tends to 0 for $\widetilde{c} \rightarrow 0^{+}$and converges to 1 as $\widetilde{c} \rightarrow+\infty$. In addition, if $\widetilde{z}(\widetilde{c}, \tau) \in \Phi_{\mathbb{H}}$, then its inverse function $\widetilde{c}(\widetilde{z}, \tau)$ is a conformal function, hence matrix monotone of any order in $(0,+\infty)$. This implies (see Donoghue $1974 \mathrm{pg} .86)$ that the derivative function $\widetilde{c}_{z}(\widetilde{z}, t)$ is completely monotone, i.e. $(-1)^{n} \frac{d^{n}}{d z^{n}} \widetilde{c}_{z}(\widetilde{z}, \tau) \geq 0, \forall n \geq 1$, hence $\widetilde{c}_{z z}(\widetilde{z}, \tau)<0$ and $\widetilde{c}_{z}$ is decreasing. The ratio $\widetilde{z} / \widetilde{c}(\widetilde{z}, \tau)$ is increasing when $E_{\widetilde{z}}<1$, decreasing when $E_{\widetilde{z}}>1$, in fact:

$$
\widetilde{z}\left[\frac{\widetilde{c}(\widetilde{z}, \tau)}{\widetilde{z}}\right]^{-1} \frac{d}{d \widetilde{z}} \frac{\widetilde{c}(\widetilde{z}, \tau)}{\widetilde{z}}=\frac{\widetilde{z} c_{z}(\widetilde{z}, \tau)}{\widetilde{c}(\widetilde{z}, \tau)}-1=E_{\widetilde{z}}-1
$$

In conclusion the coefficient $E_{\widetilde{z}}$ is positive, it is the product of an decreasing function multiplied by a function increasing in the region $E_{\widetilde{z}}<1$, and decreasing in the region $E_{\widetilde{z}}>1$. Hence $\lim _{\widetilde{c} \rightarrow 0^{+}} E_{\widetilde{z}}=0^{+}, E_{\widetilde{z}}$ is positive and cannot increase beyond 1 , then we can conclude that it is bounded.

Having proved that the time change density $\nu$ is bounded, we have shown that the family $\widetilde{z}(\widetilde{c}, \tau)$ solves a martingale problem for a time changed Bessel process, with initial condition in $\Phi_{\mathbb{H}}$, and it is possible to conclude $\widetilde{z}(\widetilde{c}, \tau) \in$ $\Phi_{\mathbb{H}}, \forall \tau \in[0, T]$.

\section{Verification of Condition A}

Notice that $\widetilde{z}(\widetilde{c}, \tau) \in \Phi_{\mathbb{H}}$ is the inverse of a conformal function which is matrix monotone, hence it is monotonic increasing and infinitely differentiable with respect to $\widetilde{c}$ and first order differentiable with respect to time. These properties imply that $\widetilde{V}(y, t)$ has all the regularity properties claimed in Condition A. In addition the coefficient $\widetilde{R}(y, t)$ is bounded by $\gamma$ :

$$
\widetilde{R}(y, t) \triangleq \frac{-\widetilde{V}_{y}(y, t)}{y \widetilde{V}_{y y}(y, t)}=\gamma \frac{\widetilde{z}(\widetilde{c}, \tau)}{\widetilde{c}_{\widetilde{c}}(\widetilde{c}, \tau)}<\gamma
$$

Given the above conclusions, Condition $\mathrm{A}$ is verified and application of Proposition 1 determines uniquely a convex $\widetilde{V}(y, t)$ and by duality $V(z, t)$ which is the unique solution to the reduced HJB. Then $V(l, h, t)=$ $h^{1-\gamma} V(z, t)$ solves the original stochastic optimization problem. 


\subsection{Numerical determination of the dual value func- tion and of its domain.}

We will show that after the insertion of eq.(22), the HJB equation (16) becomes equivalent to a system of linear ODE with respect to time.

\subsubsection{The zero order term}

Identifying the terms proportional to $y^{b}$ in the HJB and observing that $\left(y \frac{\partial}{\partial y}\right) y^{b}=b y^{b}$, we immediately obtain that (14) implies the following ODE for $B_{0}(t)$ :

where

$$
\begin{aligned}
\frac{d}{d t} B_{0}(t) & =-a_{0} B_{0}(t)+\frac{1}{b} \\
B_{0}(T) & =-\frac{\beta^{1 / \gamma}}{b}
\end{aligned}
$$

$$
\begin{aligned}
a_{0} & =\left(K_{1}-\left(K_{2}+K_{1}\right) b+K_{3}\left(b^{2}-b\right)-K_{4} \frac{b^{2}}{b^{2}-b}\right) \\
& =\left(r+\frac{\kappa-r}{\gamma}-\frac{1}{2} \frac{(1-\gamma)}{\gamma^{2}}\left(\frac{(\alpha-r)}{\sigma}\right)^{2}\right),
\end{aligned}
$$

this term corresponds to the standard Merton solution which would be exact in absence of the illiquid asset (see equation 37). This equation is a linear non homogenous ODE and can be explicitly solved:

$$
B_{0}(t)=\left\{\exp \left[a_{0}(T-t)\right]\left(\beta^{1 / \gamma}+\frac{1}{a_{0}}\right)-\frac{1}{a_{0}}\right\}\left(-\frac{1}{b}\right) .
$$

\subsubsection{First order term}

Repeating the procedure for the terms proportional to $y$ we get an ODE for

$$
\begin{aligned}
\frac{d}{d t} B_{1}(t) & =-a_{1} B_{1}(t)-\delta \\
B_{1}(T) & =+1,
\end{aligned}
$$

where (see equation 41):

$$
\begin{aligned}
a_{1} & =\left(2 \gamma K_{4}-K_{2}\right) \\
& =-\left(+(\alpha-r) \frac{\eta \rho}{\sigma}-(\mu-\delta-r)\right),
\end{aligned}
$$

and correspondingly the solution will be

$$
B_{1}(t)=\exp \left[a_{1}(T-t)\right]\left(1+\frac{\delta}{a_{1}}\right)-\frac{\delta}{a_{1}} .
$$




\subsubsection{N-th order correction}

In this section we derive from the dual HJB a hierarchy of ODE for the coefficients $B_{n}(t)$ which can be recursively solved. As a first step we guess that the dual value function can be written as:

$$
\begin{aligned}
\widetilde{V}(y, t) & =y^{b} \phi\left(y^{1 / \gamma}, t\right) \\
\phi(x, t) & =\sum_{n=0}^{\infty} B_{n}(t) x^{n} .
\end{aligned}
$$

Now we derive the hierarchy of ODE equations for $\phi(x, t)$ obtained from the dual HJB. First of all we express the operators $y \frac{\partial}{\partial y}$ and $y^{2} \frac{\partial^{2}}{\partial y^{2}}$ in terms of the number operator $N:=x \frac{\partial}{\partial x}$ where $x=y^{1 / \gamma}$ :

$$
\begin{aligned}
y \frac{\partial}{\partial y} y^{b+n / \gamma} & =\left(b+\frac{n}{\gamma}\right) y^{b+n / \gamma}= \\
& =y^{b}\left[\left(b+x \partial_{x}\right) x^{n}\right]_{\mid x=y^{1 / \gamma}} \\
& =y^{b}\left[C_{N} x^{n}\right]_{\mid x=y^{1 / \gamma}} \\
C_{N} & :=b+N \\
y^{2} \frac{\partial}{\partial y^{2}} y^{b+n / \gamma} & =\left[\left(b+\frac{n}{\gamma}\right)^{2}-\left(b+\frac{n}{\gamma}\right)\right] y^{b+n / \gamma} \\
& =y^{b}\left[D_{N} x^{n}\right]_{\mid x=y^{1 / \gamma}}, \\
D_{N} & :=\left(b+\frac{N}{\gamma}\right)\left(b+\frac{N}{\gamma}-1\right)
\end{aligned}
$$

Inserting the guess solution $y^{b} \phi\left(y^{1 / \gamma}, t\right)$ in the homogeneous version of the dual HJB and factoring the term $y^{2 b}$ it is immediate to verify that the equation for $\phi(x, t)$ can be rewritten in terms of the number operator $N$ as:

$$
\begin{aligned}
& {\left[\phi_{t}(x, t)+K_{1} \phi(x, t)-\left(K_{1}+K_{2}\right) C_{N} \phi(x, t)\right] D_{N} \phi(x, t)+} \\
& +\left[K_{3} D_{N} \phi(x, t)-\frac{1}{b}+\delta x\right] D_{N} \phi(x, t)-K_{4}\left[C_{N} \phi(x, t)\right]^{2}=0
\end{aligned}
$$

Then the ODE flow can now be uniquely determined by the PDE inserting the series expansion:

$$
\phi(x)=\sum_{n=0}^{+\infty} B_{n}(t) x^{n}
$$


in eq.(61) and imposing that the coefficient of the monomial $x^{m}$ vanishes for any $m$ :

$$
\begin{aligned}
0= & \left\langle x^{m},\left[\phi_{t}(x, t)+K_{1} \phi(x, t)-\left(K_{1}+K_{2}\right) C_{N} \phi(x, t)\right] D_{N} \phi(x, t)\right\rangle+ \\
& +\left\langle x^{m},\left[K_{3} D_{N} \phi(x, t)-\frac{1}{b}+\delta x\right] D_{N} \phi(x, t)-K_{4}\left[C_{N} \phi(x, t)\right]^{2}\right\rangle .
\end{aligned}
$$

Correspondingly the $m-t h$ order equation will be:

$$
\begin{aligned}
& H_{m}=\sum_{n=0}^{m} D_{m-n} B_{m-n}(t) \partial B_{n}(t) / \partial t+ \\
& +\sum_{n=0}^{m} D_{m-n} B_{m-n}(t)\left(K_{1}-\left(f K_{2}+K_{1}\right) C_{n}+K_{3} D_{n}\right) B_{n}(t)+ \\
& +\sum_{n=0}^{m} D_{m-n} B_{m-n}(t)\left[\delta \Delta_{1, n}-\frac{1}{b} \Delta_{0, n}\right]+ \\
& -K_{4} \sum_{n=0}^{m} C_{m-n} B_{m-n}(t) C_{n} B_{n}(t),
\end{aligned}
$$

where $\Delta_{i, j}$ denotes the Kronecker symbol, defined by: $\Delta_{i, j}=1$ if $i=j$, $\Delta_{i, j}=0$ if $i \neq j$. The infinite hierarchy of ODE equations $H_{m}=0, m \in \mathbb{N}$, can be put in normal form as stated in the following

Proposition $11 \forall N \in \mathbb{N}$, the system of $O D E$ equations:

$$
H_{m}=0, m=0,1,2, . ., N
$$

is equivalent to the set of equations:

$$
\begin{aligned}
0= & d B_{n}(t) / d t+\left(K_{1}-\left(K_{2}+K_{1}\right) C_{n}+K_{3} D_{n}\right) B_{n}(t)+\delta \Delta_{1, n}-\frac{1}{b} \Delta_{0, n} \\
& -K_{4} \sum_{k, m=0}^{N} M_{n+1, m+1}^{(N)} G_{m-k}^{(N)} v_{k+1}^{(N)} \quad n \geq 0,
\end{aligned}
$$

where:

$$
\begin{array}{rlll}
M^{(N)} & : & =\left(T p^{(N)}\right)^{-1} \\
T p_{n+1, l+1}^{(N)} & : & =D_{n-l} B_{n-l}(t), n \geq l & T p_{n+1, l+1}^{(N)}=0, \quad n<l, \\
G_{n+1, l+1}^{(N)} & : \quad=C_{n-l} B_{n-l}(t), n \geq l & G_{n+1, l+1}^{(N)}=0, \quad n<l, \\
T p^{(N)}, G^{(N)} & \in & M(\mathbb{R})_{(N+1) \times(N+1)} \\
v_{l+1}^{(N)} & :=C_{l} B_{l}(t), \quad l=0, \ldots, N .
\end{array}
$$


Proof Note that in matrix-vector notation the system of ODE equations can be written like:

$$
T p^{(N)} \cdot w^{(N)}-K_{4} G^{(N)} \cdot v^{(N)}=0,
$$

where $\cdot$ denotes the matrix product and:

$$
\begin{aligned}
w_{n+1}^{(N)} & =\partial B_{n}(t) / \partial t+\left(K_{1}-\left(K_{2}+K_{1}\right) C_{n}+K_{3} D_{n}\right) B_{n}(t)+\delta \Delta_{1, n}-\frac{1}{b} \Delta_{0, n} \\
n & =0, . ., N .
\end{aligned}
$$

$T p^{(N)}$ is an invertible matrix, in fact it is triangular and along the main diagonal $T P_{i, i}=D_{0} B_{0}(t)$ which is different from 0 for any $t \in[0, T]$. Hence left multiplying (62) by $M^{(N)}=\left(T p^{(N)}\right)^{-1}$ we get:

$$
\begin{aligned}
M^{(N)} \cdot T p^{(N)} \cdot w^{(N)}-K_{4} M^{(N)} \cdot G^{(N)} \cdot v^{(N)} & = \\
w^{(N)}-K_{4} M^{(N)} \cdot G^{(N)} \cdot v^{(N)} & =0,
\end{aligned}
$$

and the claim follows.

Note that the equations for $N=0$ and $N=1$ correspond to those obtained in the previous sections. If we consider the equation for the coefficient of order $N \geq 2$, then the equation to be satisfied by $B_{N}(t)$ is given by:

$$
\begin{gathered}
0=\left[d B_{N}(t) / d t+\left[K_{1}-\left(K_{2}+K_{1}\right) C_{N}+K_{3} D_{N}\right] B_{N}(t)\right] \\
-K_{4} \sum_{m, l=0}^{N} M_{N+1, m+1}^{(N)} G_{m+1, l+1}^{(N)} C_{l} B_{l}, \\
B_{0}(T)=-1 / b, \quad B_{1}(T)=1, \quad B_{n}(T)=0 \quad 2 \leq n \leq N .
\end{gathered}
$$

This system of equations is highly non linear due to the last term.

A crucial property is that the set of ODE can be recursively solved. In fact, consider the ODE equation to be satisfied by the highest order coefficient $B_{N}(t)$ given the coefficients $\left\{B_{n}(t)\right\}_{n=0, \ldots, N-1}$ : this is a linear equation in $B_{N}(t)$ which does not involve higher order coefficients $B_{N^{\prime}}(t), N^{\prime}>N$. In fact at level $N \geq 2$ the equation will be:

$$
\begin{aligned}
0= & {\left[d B_{N}(t) / d t+\left(K_{1}-\left(K_{2}+K_{1}\right) C_{N}+K_{3} D_{N}\right) B_{N}(t)\right] } \\
& -K_{4} \sum_{m, l=0}^{N} M_{N+1, m+1}^{(N)} G_{m+1, l+1}^{(N)} v_{l+1}^{(N)},
\end{aligned}
$$


and extracting from the last summation the terms which contain $B_{N}$ we get:

$$
\begin{array}{rl}
0= & d B_{N}(t) / d t-K_{4} f^{(N)}(t)+ \\
& {\left[K_{1}-\left(K_{2}+K_{1}+\frac{2 K_{4}}{b-1}\right) C_{N}+\left(K_{3}+\frac{K_{4}}{(b-1)^{2}}\right) D_{N}\right] B_{N}(t),} \\
f^{(N)}(t)= & \sum_{m=0}^{N} \sum_{l=0}^{N} M_{N+1, m+1}^{(N) *} G_{m+1, l+1}^{(N) *} v_{l+1}^{(N) *}, \\
M^{(N) *}= & \left(T p^{(N) *}\right)^{-1}, \\
(T p)_{N+1,1}^{(N) *} & 0,(T p)_{m+1, n+1}^{(N) *}=(T p)_{m+1, n+1} \quad(m, n) \neq(N, 0) \\
G_{N+1,1}^{(N) *}= & 0, G_{m+1, n+1}^{(N) *}=G_{m+1, n+1}^{(N)} \quad(m, n) \neq(N, 0) \\
v_{N+1}^{(N) *}= & 0, v_{l+1}^{(N) *}=C_{l} B_{l} \quad l \neq N .
\end{array}
$$

Then the linear ODE to be solved is:

$$
\begin{aligned}
\partial B_{N}(t) / \partial t & =-a_{N} B_{N}(t)+K_{4} f^{(N)}(t) \\
B_{N}(T) & =0,
\end{aligned}
$$

where

$$
a_{N}=\left[K_{1}-\left(K_{2}+K_{1}+\frac{2 K_{4}}{b-1}\right) C_{N}+\left(K_{3}+\frac{K_{4}}{(b-1)^{2}}\right) D_{N}\right],
$$

and

$$
f^{(N)}(t)=\sum_{m=0}^{N} \sum_{l=0}^{N} M_{N+1, m+1}^{(N) *} G_{m+1, l+1}^{(N) *} v_{l+1}^{(N) *} .
$$

The general solution to this equation will be:

$$
B_{N}(t)=-K_{4} \int_{t}^{T} e^{a_{N}(s-t)} f^{(N)}(s) d s .
$$

In order to identify the solution it is necessary to impose the boundary conditions. This can be easily done imposing the condition that the Taylor coefficients of the function $z(c, t)$ expanded around $c_{B}(t)$ are zero.

\subsection{Accuracy of the numerical determination of the dual value function.}

In the region of convergence the determination of $\widetilde{V}(y, 20)$ is largely independent from $N$ for $N>10$. The coefficients of the series converge to zero exponentially fast. Within the numerical accuracy of double precision, the value of the numerical determination is independent from the truncation order. 


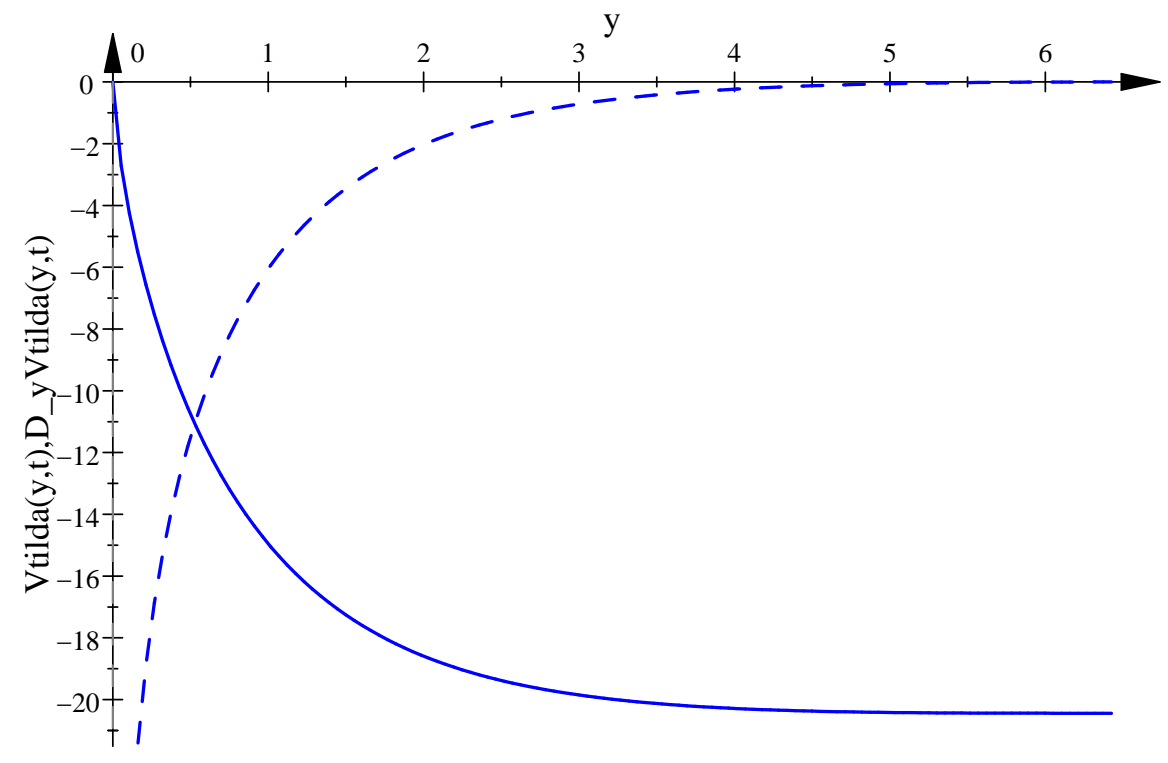

Figure 10: Dual Value function and its derivative with respect to y (dotted line)

\subsection{Computation of partial derivatives}

$$
\begin{aligned}
V_{t}(l, h, t) & =-\kappa h^{1-\gamma} V(z, t)+h^{1-\gamma} V_{t}(z, t), \\
V(l, h, t) & =h^{1-\gamma} V(z, t), \\
V_{l}(l, h, t) & =h^{-\gamma} V_{z}(z, t), \\
V_{l l}(l, h, t) & =h^{-\gamma-1} V_{z z}(z, t), \\
V_{h}(l, h, t) & =h^{-\gamma}\left[(1-\gamma) V(z, t)-z V_{z}(z, t)\right], \\
V_{h h}(l, h, t) & =h^{-\gamma-1}\left[-\gamma(1-\gamma) V(z, t)+2 \gamma z V_{z}(z, t)+z^{2} V_{z z}(z, t)\right], \\
V_{l h}(l, h, t) & =-h^{-\gamma-1}\left[\gamma V_{z}(z, t)+z V_{z z}(z, t)\right] .
\end{aligned}
$$

\subsection{Proof of equation (36)}

If we define $q$ as:

$$
q:=\left[\frac{(1-\gamma) V(l, h, t)}{h^{1-\gamma}(z+p(z, t))^{1-\gamma}}\right]^{-1 / \gamma}
$$




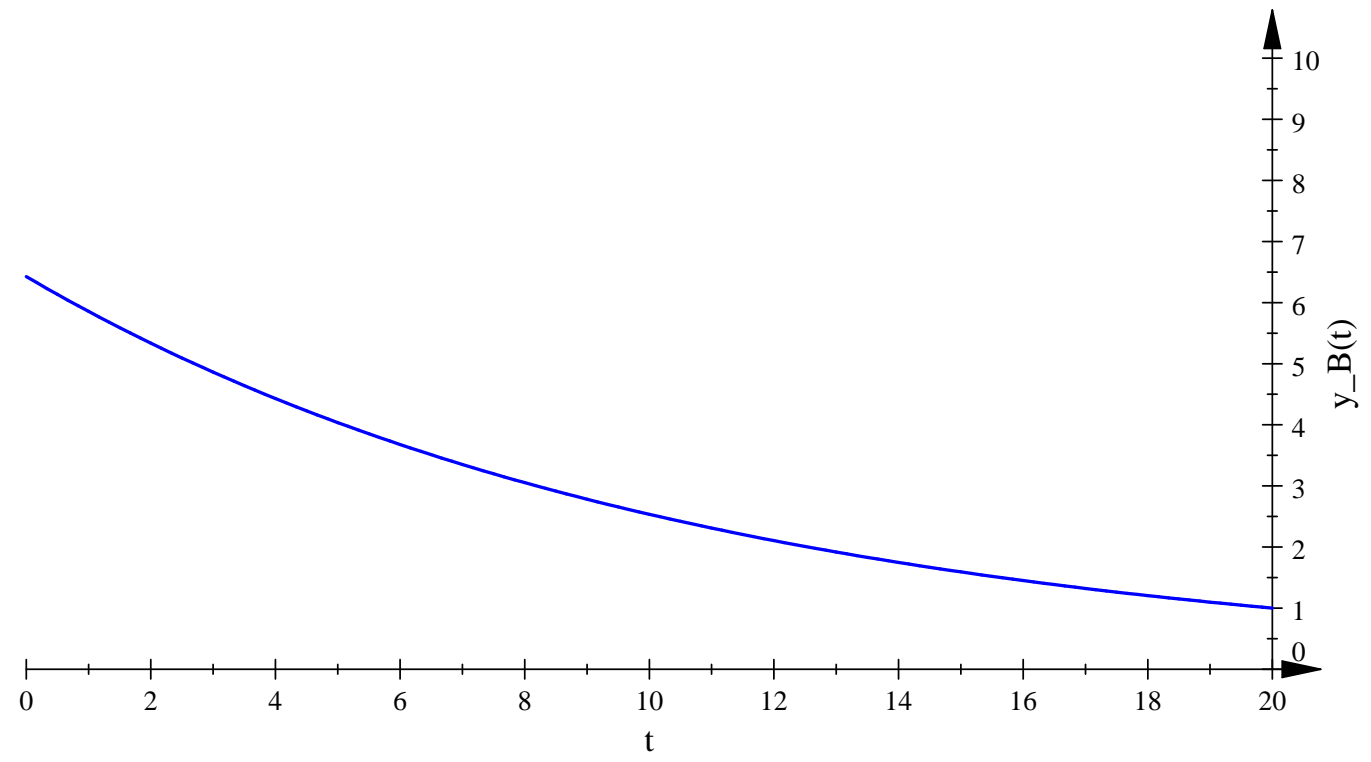

Figure 11: A numerical determination of $y_{B}(t)$ under the parametric conditions given in Table 1.

homogeneity of $V$ implies that $q$ is a function of $z$ and $t$ only, hence we can represent $V(l, h, t)$ and $V(z, t)$ as:

$$
\begin{aligned}
V(l, h, t) & =q(z, t)^{-\gamma} \frac{w(l, h, t)^{1-\gamma}}{1-\gamma} \\
V(z, t) & =q(z, t)^{-\gamma} \frac{(z+p(z))^{1-\gamma}}{1-\gamma} .
\end{aligned}
$$

By eq. (7) optimal consumption is given by:

$$
c^{*}(l, h, t)^{-\gamma}=V_{l}(l, h, t)=h^{-\gamma} V_{z}(z, t),
$$

and by the definition of $p(z, t)$ :

$$
V_{z}(z, t)=(1-\gamma) \frac{V(z, t)}{z+p(z)}=q(z, t)^{-\gamma}(z+p(z))^{-\gamma},
$$

hence the consumption over wealth ratio will be defined by:

$$
\begin{aligned}
\frac{c^{*}(l, h, t)}{w(l, h, t)} & =\frac{\left(V_{z}(z, t)\right)^{-1 / \gamma}}{z+p(z)} \\
& =\frac{\left(q(z, t)^{-\gamma}(z+p(z))^{-\gamma}\right)^{-1 / \gamma}}{z+p(z)} \\
& =q(z, t) .
\end{aligned}
$$




\subsection{Proof of equation (38)}

The maximization in eqs.(8) yields the risky allocation formula:

$$
-\frac{V_{l}(l, h, t)}{V_{l l}(l, h, t)} \frac{\alpha-r}{\sigma^{2}}-\frac{h V_{l h}(l, h, t)}{V_{l l}(l, h, t)} \frac{\eta \rho}{\sigma},
$$

and applying eqs.(66) we obtain:

$$
\left[\frac{\alpha-r-\gamma \eta \rho \sigma}{\sigma^{2}}\left(-\frac{V_{z}(z, t)}{V_{z z}(z, t)}\right)-\frac{\eta \rho}{\sigma} z\right] h,
$$

and using the definition of $R(z, t)$ we conclude.

\subsection{Proof of Corollary 6}

The proof is an immediate consequence of He and Pearson (1991) Th.7 and similar to Proposition 1 in Teplà (2000). Th.7 states the following: let $l(\lambda, h, t)$ be the liquid wealth considered as a function of $h, t$ and $\lambda=V_{l}(l, h, t)$, the conjugate variable of $l$, then following feedback representation for the the volatility of the SDF, i.e. optimal risk adjustment on $W_{t}^{2}, \widetilde{\sigma}_{2}^{S D F}$, holds:

$$
\widetilde{\sigma}_{2}^{S D F}(\lambda, h, t)=\frac{h l_{h}(\lambda, h, t)}{\lambda l_{\lambda}(\lambda, h, t)} \eta\left(1-\rho^{2}\right)^{1 / 2}
$$

Since we apply duality to the reduced value function $V(z, t)$, and to the rescaled liquid wealth $z=l / h$, it is straightforward to prove that homogeneity implies the relation: $\lambda=y h^{-\gamma}$. In terms of reduced variable $y$ and $h$, the feedback formula for $\tilde{\sigma}_{2}^{S D F}$ will be:

$$
\tilde{\sigma}_{2}^{S D F}(\lambda(y, h), h, t)=\left[\gamma-\frac{l(\lambda(y, h), h, t)}{y l_{y}(\lambda(y, h), h, t)}\right] \eta\left(1-\rho^{2}\right)^{1 / 2}
$$

Since $l(\lambda(y, h), h, t)=h z(y, t)$ where $z(y, t)=-\widetilde{V}_{y}(y, t)$ then we can conclude that:

$$
\sigma_{2}^{S D F}(y, t)=\widetilde{\sigma}_{2}^{S D F}(y, 1, t)=\left(\gamma-\frac{\widetilde{V}_{y}(y, t)}{y \widetilde{V}_{y y}(y, t)}\right) \eta\left(1-\rho^{2}\right)^{1 / 2},
$$

and (40) holds. 


\subsection{Proof of equation (42)}

Optimal consumption is given by (7) hence: $c^{*}(l, h, t)=\left[V_{l}(l, h, t)\right]^{-1 / \gamma}$. In terms of the state variables $h, z$ and $t$ we have:

$$
\begin{aligned}
l(h, z, t) & =h z \\
c^{*}(l(z, h, t), h, t) & =h V_{z}(z, t)^{-1 / \gamma} .
\end{aligned}
$$

Then the elasticity of consumption w.r.t liquid wealth can be written as:

$$
\begin{aligned}
\frac{l(z, h, t)}{c^{*}(l(z, h, t), h, t)} \frac{\partial c^{*}((z, h, t), h, t)}{\partial l(z, h, t)}= & \frac{z h}{h V_{z}(z, t)^{-1 / \gamma}} \frac{\partial\left(h V_{z}(z, t)^{-1 / \gamma}\right)}{\partial z} \frac{\partial z}{\partial l(z, h, t)} \\
& +\frac{l}{h V_{z}(z, t)^{-1 / \gamma}} \frac{\partial\left(h V_{z}(z, t)^{-1 / \gamma}\right)}{\partial h} \frac{\partial h}{\partial l(z, h, t)},
\end{aligned}
$$

which equals

$$
\frac{l}{c^{*}(l, h, t)} \frac{\partial c^{*}(l, h, t)}{\partial l(l, h, t)}=-\frac{1}{\gamma} \frac{z V_{z z}(z, t)}{V_{z}(z, t)}+\frac{h}{c^{*}(l, h, t)} \frac{\partial c^{*}(l, h, t)}{\partial h},
$$

hence by eq.(39) we can conclude:

$$
\frac{l}{c^{*}(l, h, t)} \frac{\partial c^{*}(l, h, t)}{\partial l}-\frac{h}{c^{*}(l, h, t)} \frac{\partial c^{*}(l, h, t)}{\partial h}=\frac{R(z)}{\gamma} .
$$

